\title{
TRATAMIENTO MÉDICO E INTERVENCIONISTA DE LOS PACIENTES DIABÉTICOS CON SINDROME CORONARIO AGUDO.
}

\author{
Doctorando: Anna Baeza Román. \\ Director de Tesis: Dr. Jaime Latour Pérez.
}

PROGRAMA DE DOCTORADO EN CIENCIAS DE LA SALUD DE LA UNIVERSIDAD JAUME I DE CASTELLÓN.

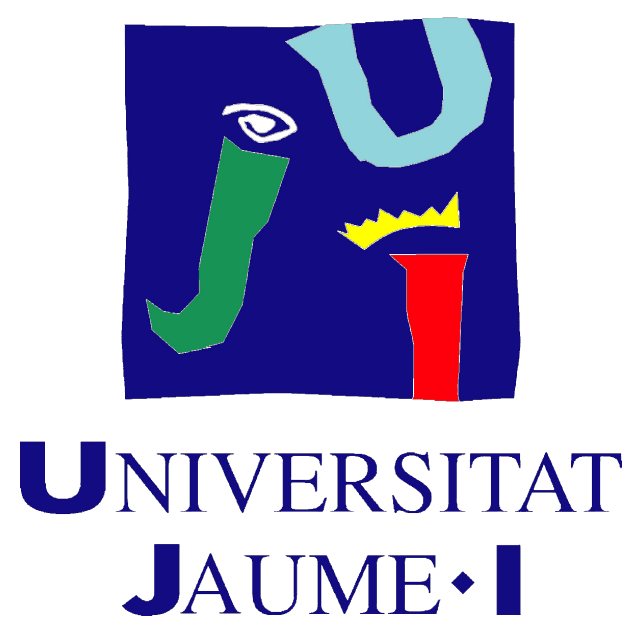


PROGRAMA DE DOCTORADO EN CIENCIAS DE LA SALUD DE LA UNIVERSIDAD JAUME I DE CASTELLÓN.

Admisión al periodo investigador: 18 de octubre de 2011 .

TÍTULO: TRATAMIENTO MÉDICO E INTERVENCIONISTA DE LOS PACIENTES DIABÉTICOS CON SÍNDROME CORONARIO AGUDO.

RESPONSABLES DEL PROYECTO:

- Doctorando: Anna Baeza Román.

○ Licenciada en Medicina y Cirugía. Universidad Miguel Hernández de Elche (Junio 2005).

○ Especialista en Medicina Intensiva (Mayo 2012). Hospital General Universitario de Elche.

- Director y asesor de metodología: Dr. Jaime Latour Pérez

- Especialista en Medicina Intensiva. Hospital General Universitario de Elche

○ Departamento de Medicina Clínica. Universidad Miguel Hernández de Elche.

- Ámbito de desarrollo: Unidad de Cuidados Intensivos y Coronarios. Hospital General Universitario de Elche. Camí de la Almazara $n^{\circ} 11.03203$ Elche (Alicante). 


\section{Agradecimientos...}

- A mi director de Tesis, Jaime, por su confianza, orientación y paciencia. Y por saber contagiarnos de su pasión por la investigación.

- A Eva, compañera de batallas, sufridora de la base de datos. Por su gran apoyo, dentro y fuera de este trabajo.

- A mis padres, siempre dispuestos, de forma incondicional.

- A mi pequeña familia, Xavi y Laia. 


\section{ÍNDICE:}

1. Principales abreviaturas.

2. Introducción general.

3. Objetivos.

4. Metodología general.

5. Resumen global de los resultados y discusión de los mismos.

6. Discusión general.

7. Conclusiones.

8. Bibliografía.

9. Anexo 1: Hospitales participantes en el registro ARIAM-SEMICYUC.

10. Anexo 2: Factor de impacto y área temática de las revistas.

11. Anexo 3: Artículos.
a. Artículo 1 .
b. Artículo 2 .
c. Carta al editor y replica de los autores I.
d. Carta al editor y replica de los autores II.
e. Estudio 3. 


\section{PRINCIPALES ABREVIATURAS UTILIZADAS EN EL TEXTO:}

\begin{tabular}{|l|l|}
\hline ARIAM & Análisis del Retraso en el infarto Agudo de Miocardio \\
\hline CI & Cardiopatía isquémica \\
\hline CRUSADE & $\begin{array}{l}\text { Can Rapid risk stratification of Unstable angina patients Suppress } \\
\text { ADverse outcomes with Early implementation of the ACC/AHA } \\
\text { Guidelines. }\end{array}$ \\
\hline DLP & Dislipemia \\
\hline DM & Diabetes mellitus \\
\hline ECG & Electrocardiograma \\
\hline GRACE & Global Registry of Acute Coronary Events. \\
\hline HTA & Hipertensión arterial \\
\hline IAM & Infarto agudo de miocardio \\
\hline ICC & Insuficiencia cardiaca congestiva \\
\hline ICP & Intervencionismo percutáneo \\
\hline KK & Killip-Kimball \\
\hline PCM & Primer contacto médico \\
\hline RCP & Resucitación cardiopulmonar \\
\hline SCA & Sindrome coronario agudo \\
\hline SCACEST & Sindrome coronario agudo con elevación del segmento ST. \\
\hline SCASEST & Soronarias. \\
\hline SEMICYUC & The Thrombonario agudo sin elevación del segmento ST. \\
\hline UCI & Sing cuidados intensivos \\
\hline
\end{tabular}


Las enfermedades cardiovasculares son actualmente la principal causa de muerte en los países desarrollados, siendo la enfermedad coronaria la manifestación más frecuente, asociándose con una alta morbi-mortalidad. En nuestro país, la cardiopatía isquémica (CI) ocasiona el mayor número de muertes cardiovasculares (30\% del total, un $37 \%$ en varones y un $24 \%$ en las mujeres). Estos datos la sitúan como la causa principal de muerte en el $12 \%$ de los varones y el $10 \%$ de las mujeres españolas, y su tasa de morbilidad hospitalaria es de 352 casos por 100.000 habitantes (493 en varones y 215 en mujeres $)^{[1][2]}$. Por tanto, la cardiopatía isquémica genera una gran demanda asistencial con el consiguiente consumo de recursos.

Aproximadamente un $20-35 \%$ de los pacientes con síndrome coronario agudo (SCA) tiene diabetes mellitus (DM) conocida, y al menos la misma proporción tiene diabetes no diagnosticada o intolerancia a la glucosa. En el registro español ARIAMSEMICYUC del año 2015, la prevalencia de diabetes es del 23,8\% en los paciente con síndrome coronario agudo con elevación del segmento ST (SCACEST), y del 33,8\% en los pacientes con síndrome coronario agudo sin elevación del segmento ST $(\text { SCASEST })^{[3]}$. Además, la diabetes es un predictor independiente de mortalidad e infarto agudo de miocardio (IAM) no letal ${ }^{[4][5]}$. Los pacientes diabéticos con SCA son mayores, predominantemente mujeres, tienen más comorbilidad como la hipertensión (HTA), dislipemia (DLP) e insuficiencia renal, con más frecuencia se presentan con síntomas atípicos, y son más propensos a desarrollar complicaciones, especialmente insuficiencia cardíaca y hemorragias ${ }^{[4][6]}$.

Las principales guías del manejo del SCA (con y sin elevación de ST) consideran al paciente diabético con SCA como paciente de alto riesgo y recomiendan un manejo agresivo farmacológico e intervencionista, similar a los pacientes de alto riesgo no diabéticos ${ }^{[7][8][9][10][11]}$. Sin embargo, diferentes registros han mostrado una infrautilización del tratamiento basado en la evidencia, tanto farmacológico como de revascularización en los pacientes diabéticos ${ }^{[5][6][12][13][14]}$.

Por otro lado, a pesar de los avances en el manejo del SCA, no se ha encontrado una reducción significativa de la mortalidad respecto a los pacientes no diabéticos ${ }^{[15][16]}$. Los pacientes diabéticos siguen presentado una mayor tasa de mortalidad por todas las causas, así como una mayor tasa de complicaciones y eventos cardiovasculares que los 
pacientes no diabéticos ${ }^{[17][18][19]}$.

Existe cierta incertidumbre sobre las causas de esta peor evolución. En primer lugar, las características basales de los pacientes con diabetes difieren de las de aquellos pacientes que no la padecen, por lo que es posible, que estas diferencias (mayor edad, mayor prevalencia de mujeres, la diferente comorbilidad...) puedan ser variables asociadas a sus peores resultados ${ }^{[20]}$. Por otro lado, la diferente forma de presentación del SCA en estos pacientes puede prolongar el tiempo de ingreso en el hospital, provocando un retraso en el diagnóstico y reduciendo de este modo la oportunidad de administrar un tratamiento adecuado ${ }^{[21]}$. En tercer lugar, la infrautilización del tratamiento basado en la evidencia puede estar jugando un papel importante, sin conocer los motivos de este enfoque mas conservador en estos pacientes ${ }^{[12]}$. Por último, los pacientes diabéticos tienen una mayor tasa de enfermedad multivaso. La severidad de la enfermedad de las arterias coronarias puede ser un determinante importante para su supervivencia a largo plazo $^{[21][22][23]}$. 


\section{OBJETIVOS :}

De todas estas incertidumbres descritas, surgen una serie de hipótesis sobre las que recae el marco conceptual de este trabajo:

Hipótesis 1: El tratamiento de los pacientes diabéticos con SCA es excesivamente conservador en relación con las recomendaciones de las principales guías clínicas.

Hipótesis 2: El exceso de mortalidad de los pacientes diabéticos con SCA se debe, al menos en parte, al menor esfuerzo terapéutico relativo a la gravedad.

Hipótesis 3: Existen diferentes determinantes que explican el mayor uso de una estrategia conservadora en los pacientes diabéticos con SCASEST.

Hipótesis 4: Los pacientes diabéticos con SCACEST no acuden al sistema sanitario de la misma forma ni con la misma rapidez desde la aparición de los síntomas que los pacientes no diabéticos.

Hipótesis 5: El retraso en la accesibilidad de los pacientes diabéticos puede influir en la mayor mortalidad de estos pacientes.

Hipotesis 6: La principal escala de riesgo isquémico, el score GRACE, no tiene el mismo poder predictivo para muerte intrahospitalaria en los pacientes diabéticos.

Las 2 primeras hipótesis se han confirmado previamente en diversos estudios publicados. Por un lado, ya se ha comentado previamente que existe una infrautilización del tratamiento basado en la evidencia, tanto farmacológico como de revascularización en estos pacientes ${ }^{[12]}$. Por otro lado existe evidencia de que el uso de la estrategia invasiva en pacientes de alto riesgo, entre los que se incluyen los pacientes diabéticos reducen la mortalidad ${ }^{[13][24][25][26][27]}$. Sin embargo, no se conoce bien el perfil específico de los pacientes diabéticos que se manejan de forma conservadora, por lo que la primera parte de este trabajo se centrará en confirmar la tercera hipótesis.

La segunda parte de este trabajo se centra en aclarar las siguientes 2 hipótesis, ya que los pacientes con SCACEST deben recibir tratamiento de reperfusión lo antes posible, y en cualquier caso en las primeras 12 horas tras el inicio de los síntomas $^{[9][10][28]}$.

Varios estudios han objetivado que los pacientes diabéticos con SCACEST tardan más en contactar con el sistema sanitario tras el inicio de los síntomas ${ }^{[29][30][31]}$, lo 
cual influirá, por un lado, en la decisión de realizar reperfusión y en la elección del tratamiento y, por otro lado, provocará un mayor tiempo de isquemia miocárdica.

Intentaremos aclarar si existen diferencias en la accesibilidad al sistema sanitario (tiempos de acceso y de reperfusión) de los pacientes diabéticos respecto a los no diabéticos, analizando los diferentes factores que pueden influenciar estas diferencias, y por último analizar si explican la mayor mortalidad de éstos.

Finalmente, la última hipótesis a estudiar (Hipótesis 6), surge durante la realizaicón del trabajo de investigación. La estratificación del riesgo en el manejo inicial de los pacientes con SCA está recomendado en las principales guías clínicas, y es ampliamente utilizado en la práctica clínica habitual, siendo los principales scores utilizados, el CRUSADE, TIMI y GRACE ${ }^{[32][33][34][35]}$. Teniendo en cuenta que, como ya se ha comentado, la diabetes mellitus es una variable asociada de forma independiente con la mortalidad de los pacientes con $\mathrm{SCA}^{[4][5]}$, parece lógico que dicha variable esté incluido en estos scores. Así es en el caso del CRUSADE y TIMI, pero no en el caso del GRACE, por lo que es posible que este score no tenga el mismo poder predictivo en los pacientes diabéticos.

\section{Preguntas de investigación:}

El objetivo de este trabajo, por tanto, es responder a las siguientes preguntas de investigación:

Pregunta 1: ¿Cuales son los determinantes del uso de la estrategia invasiva precoz en los pacientes diabéticos con SCASEST?

Pregunta 2: ¿Los pacientes diabéticos con SCACEST acuden al sistema sanitario de la misma forma que los pacientes no diabéticos?

Pregunta 3: ¿Estas diferencias influyen en la mortalidad hospitalaria?

Pregunta 4: ¿La escala GRACE predice peor la mortalidad en los pacientes diabéticos? 
Preguntas de investigación en formato PICO:

\begin{tabular}{|c|c|c|c|c|}
\hline & P (Pacientes) & I (Intervención) & C (Comparación) & 0 (Outcomes) \\
\hline 1 & $\begin{array}{l}\text { Pacientes } \\
\text { diabéticos con } \\
\text { SCASEST }\end{array}$ & $\begin{array}{l}\text { Variables: Edad, } \\
\text { Sexo, Tto previo, } \\
\text { Presentación, } \\
\text { Comorbilidad, } \\
\text { Gravedad }\end{array}$ & No procede & Estrategia invasiva \\
\hline 2 & $\begin{array}{l}\text { Pacientes con } \\
\text { SCACEST }\end{array}$ & DM & No DM & $\begin{array}{l}\text { Acceso al sistema sanitario: } \\
\text { Forma, Tiempo }\end{array}$ \\
\hline 3 & $\begin{array}{l}\text { Pacientes con } \\
\text { SCACEST }\end{array}$ & $\begin{array}{l}\text { DM con retraso } \\
\text { hospitalario. }\end{array}$ & DM sin retraso hospitalario & Mortalidad hospitalaria. \\
\hline 4 & $\begin{array}{l}\text { Pacientes con } \\
\text { SCA }\end{array}$ & DM & No DM & $\begin{array}{l}\text { Mortalidad hospitalaria } \\
\text { predicha por score GRACE }\end{array}$ \\
\hline
\end{tabular}




\section{METODOLOGÍA GENERAL:}

El abordaje de estos proyectos de investigación se ha realizado mediante investigación primaria, con análisis de cohortes retrospectivo a partir de los datos del registro ARIAM-SEMICYUC.

Los datos se han analizado con SPSS Statistics para Windows versión 19.0 y con Stata versión 13. Aunque en cada unos de los artículos se especifica la metodología utilizada, de manera general, en la estadística descriptiva las variables cuantitativas continuas se han expresado como la mediana y percentiles 25 y 75, y las variables categóricas como proporciones. La comparación de variables categóricas se realiza con el test ji al cuadrado y la comparación de las variables cuantitativas mediante el test de Mann-Whitney (para comparación de 2 grupos) o de Kruskal-Wallis (para comparación de 3 o más grupos). Previamente habremos comprobado que estas variables no siguen una distribución normal mediante la prueba de Shapiro-Wilks. En el caso de análisis de tiempos de acceso, el contraste de hipótesis se realizará mediante el test del log-rank. El análisis de contraste se realizará de forma bilateral con un nivel de significación alfa (error tipo I) del $5 \%$.

El control de los factores de confusión (confounding) se realizará mediante análisis estratificado en función de las variables de confusión o análisis de regresión logística ${ }^{[36]}$.

\section{Registro ARIAM-SEMICYUC ${ }^{[3]}$ :}

El Proyecto ARIAM (Análisis del Retraso en el Infarto Agudo de Miocardio) nació en el año 1994 en Andalucía como un instrumento de mejora de calidad orientado inicialmente a acortar los tiempos de la trombolisis. Desde entonces, el registro ha evolucionado, adaptándose a los nuevos estándares del tratamiento del SCA.

Desde el año 2010 el registro ARIAM-SEMICYUC es propiedad de SEMICYUC y está integrado en el Grupo de Trabajo de Cuidados Intensivos Cardiológicos y RCP (GTCIC y RCP). En este periodo se ha pasado de un registro continuo a un registro temporal en forma de un corte anual de 3 meses.

El registro ARIAM-SEMICYUC cumple con la legislación española sobre estudios postautorización de tipo observacional para medicamentos de uso humano (Orden SAS/3470/2009, de 16 de diciembre) así como con la normativa de la Ley de 
Protección de Datos. Cuenta además con la aprobación del Comité de Ética de la Investigación del centro coordinador (Hospital Severo Ochoa de Leganés, Madrid) y ha sido autorizado localmente por cada uno de los Directores de los Centros participantes. En mayo de 2012, el registro ARIAM-SEMICYUC fue reconocido por el Ministerio de Sanidad Política Social e Igualdad como Registro de Interés para el Sistema Nacional de Salud.

Los diferentes centros hospitalarios participan de forma voluntaria y se han ido incrementando progresivamente. En el corte del año 2015 han participado un total de 69 hospitales públicos y privados de España y Andorra. Como ya se ha comentado, se recogen anualmente datos anonimizados de los pacientes en cortes trimestrales, aunque también se dispone de datos de algunos centros que mantienen el registro activo todo el año. Actualmente, disponemos de los informes de los cortes correspondientes a los años comprendidos entre 2010 y 2015.

Los criterios de inclusión en el registro son:

- Pacientes consecutivos ingresados en UCI con sospecha de SCA de menos de 48h de evolución

- $\quad$ Edad $\geq 18$ años

- Los reingresos acaecidos después de los 30 días del ingreso se consideran como nuevos casos.

- Los pacientes trasladados a otros hospitales durante la fase aguda se asignan al hospital de la primera UCI donde fueron ingresados.

El registro consta de una serie de variables (Tabla 1) ajustadas a las recomendaciones de las principales Guías de Práctica Clínica, y planes estratégicos específicos, tanto de carácter autonómico como nacional o de la Unión Europea (Documento CARDS). 
Tabla 1: Variables del registro ARIAM-SEMICYUC. Se agrupan en 7 bloques.

\begin{tabular}{|c|c|c|}
\hline 1 & Pre-UCI & $\begin{array}{lll}\text { - } & \text { Filiación y } & \text { datos } \\
& \text { sociodemográficos } & \\
\text { - } & \text { Antecedentes } \\
\text { - } & \text { Forma de Acceso a UCI } & \\
\end{array}$ \\
\hline 2 & SCACEST & - Valoración inicial \\
\hline & SCASEST & $\begin{array}{l}\text { - } \\
\text { - }\end{array}$ \\
\hline 3 & $U C I$ & $\begin{array}{ll}\text { - } & \text { Estancia } \\
\text { - } & \text { Determinaciones bioquímicas y } \\
& \text { puntuaciones } \\
\text { - } & \text { Alta de UCI }\end{array}$ \\
\hline 4 & Periodo post UCI & $\begin{array}{ll}\text { - } & \text { Alta hospitalaria } \\
\text { - } & \text { Seguimiento a los } 30 \text { días }\end{array}$ \\
\hline 5 & \multicolumn{2}{|l|}{ ICP } \\
\hline 6 & \multicolumn{2}{|l|}{ Complicaciones } \\
\hline 7 & \multicolumn{2}{|l|}{ Procedimientos } \\
\hline
\end{tabular}




\section{RESUMEN GLOBAL DE LOS RESULTADOS Y DISCUSIÓN DE LOS MISMOS.}

A continuación vamos a recuperar las preguntas de investigación que se habían planteado previamente, y las iremos contestando con los resultados obtenidos.

Pregunta 1: ¿Cuales son los determinantes del uso de la estrategia invasiva precoz en los pacientes diabéticos con SCASEST?

Para contestar esta pregunta se realiza en primer lugar un análisis univariante, incluyendo 531 pacientes diabéticos con SCASEST, de los cuales el $49.7 \%$ recibieron estrategia invasiva. Los resultados de este análisis muestran que los pacientes diabéticos manejados con estrategia invasiva precoz eran en general pacientes de menor riesgo, con las siguientes características:

- Más jóvenes

- Menos antecedentes de insuficiencia cardiaca

- Menos antecedentes de infarto de miocardio previo

- Menor prevalencia de lesiones coronarias conocidas

- Menor prevalencia de tratamiento previo con clopidogrel

- Mayor proporción de ECG de riesgo

- Menor grado de insuficiencia cardiaca medida por KK

- Menor riesgo isquémico

- Menor riesgo hemorrágico.

Posteriormente, mediante regresión logística binaria, se identifican 4 determinantes asociados de forma independiente al uso de la estrategia invasiva precoz:

1) Presencia de ECG de riesgo

2) Ausencia de tratamiento previo con clopidogrel

3) Ausencia al ingreso de Killip $>1$

4) Ausencia de riesgo hemorrágico alto 
Las guías clínicas recomiendan, efectivamente, un manejo conservador en los pacientes sin cambios severos en el ECG, lo cual se refleja en nuestros resultados. Sin embargo, recomiendan el uso de una estrategia invasiva en los pacientes de más gravedad y con mayor grado de insuficiencia cardiaca ${ }^{[7][8][11]}$.

El motivo de que los pacientes con mayor grado de ICC se manejen en nuestro estudio de forma conservadora no está claro. Como ya se ha comentado, los pacientes manejados de forma conservadora, además de presentar Killip $>1$, tenían además antecedentes de ICC e IAM previos, y mayor prevalencia de lesiones coronarias conocidas, por lo que es posible que las lesiones coronarias en este grupo fueran de mayor gravedad (enfermedad multivaso, no revascularizables...) y condicionasen esta decisión. En el registro ARIAM-SEMICYUC se recoge el antecedente de lesiones coronarias conocidas como existencia de lesiones coronarias significativas $(>50 \%)$ demostradas mediante coronariografía u otras técnicas de imagen, pero no se recoge si dichas lesiones son no revascularizables. Esto confirmaría los resultados obtenidos por otros autores, que además de objetivar igualmente una infrautilización del tratamiento de revascularización en los pacientes diabéticos con SCASEST, analizaron también los posibles motivos de dicha infrautilización, encontrando como principales causas: 1) la existencia de lesiones conocidas no revascularizables y 2) el rechazo del paciente o familia al procedimiento ${ }^{[27]}$.

Parece también, que el temor a las complicaciones hemorrágicas puede ser otra de las causas de la infrautilización de la estrategia invasiva en estos pacientes. La valoración del riesgo hemorrágico mediante el score CRUSADE es obligada puesto que la presencia de eventos hemorrágicos mayores se ha relacionado con un aumento de la mortalidad $^{[37][38]}$. Sin embargo, no existen recomendaciones claras que condicionen el uso de la estrategia invasiva en función de este riesgo hemorrágico, ni en función de la toma previa de antiagregantes o anticoagulantes orales, y que puedan justificar los hallazgos de nuestro estudio. Sí que se recomiendan diferentes estrategias para reducir el riesgo de aparición de eventos hemorrágicos en aquellos pacientes que se sometan a $\mathrm{ICP}^{[7]}$.

Finalmente, se repite el análisis de regresión logística binaria, pero esta vez incluyendo en el grupo de estrategia invasiva a aquellos pacientes sometidos a coronariografía en las primeras $72 \mathrm{~h}$. En este caso, se añade como nuevo determinante asociado al uso de la estrategia invasiva, la ausencia de tratamiento previo con acenocumarol, lo cual apoya la hipótesis de que el temor a la aparición de 
complicaciones hemorrágicas determina de forma importante la decisión del tratamiento.

Ninguno de estos resultados varía cuando se excluyen del análisis los pacientes fallecidos en las primeras $24 \mathrm{~h}$. Tampoco se ha objetivado que la presencia o no de laboratorio de hemodinámica en el hospital condicione la realización del cateterismo de forma precoz.

Pregunta 2: ¿Los pacientes diabéticos con SCACEST acuden al sistema sanitario de la misma forma que los pacientes no diabéticos?

Para contestar esta segunda pregunta, se han analizado 4685 pacientes con SCACEST, de los cuales el 22,85\% tienen DM. En el análisis univariante inicial, comparando el grupo de pacientes diabéticos respecto a los pacientes no diabéticos, no se han encontrado diferencias estadísticamente significativas respecto a la forma de acceso al sistema sanitario ni en la forma de traslado al hospital.

La principal diferencia de los pacientes diabéticos, es que éstos tardan mas en realizar el primer contacto médico, lo cual contribuye a un mayor retraso prehospitalario, y por lo tanto, de todos aquellos tiempos que se calculen a partir del inicio de los síntomas ( Tiempo síntomas-aguja; Tiempo síntomas-balón; Tiempo total de reperfusión). Sin embargo, no se han encontrado diferencias estadísticamente significativas en el resto de los tiempos que se calculan a partir del contacto con el sistema sanitario (Tiempo de traslado al hospital, Tiempo urgencias-aguja; Tiempo urgencias-balón; Tiempo PCM-aguja; Tiempo PCM-balón).

Diferentes publicaciones han mostrado resultados similares ${ }^{[5][29][31][39]}$, habiéndose identificado la DM como variable predictora independiente asociada a un retraso prehospitalario mayor a 3 horas $^{[30]}$. En nuestro estudio, los pacientes diabéticos presentan mas frecuentemente síntomas atípicos o indoloros lo cual puede explicar el mayor retraso en solicitar ayuda, hecho que también está descrito en estudios previos $^{[39][40]}$. 


\section{Pregunta 3: ¿Estas diferencias de accesibilidad al sistema sanitario de los pacientes}

diabéticos influyen en la mortalidad hospitalaria de los mismos?

El análisis univariante muestra una mayor mortalidad en UCI, hospitalaria y a los 30 días en los pacientes diabéticos. Sin embargo, el retraso atribuible al paciente no se identifica como variable predictora independiente de mortalidad en el modelo de regresión logística binaria.

Las variables identificadas como predictoras independientes de mortalidad son la diabetes y la gravedad del paciente (TIMI, GRACE y CRUSADE), pero no el retraso atribuible al paciente.

Como ya se ha comentado, publicaciones previas habían objetivado un mayor retraso prehospitalario de los pacientes diabéticos, pero en ninguna de ellas se analiza la posible relación entre este mayor retraso y su mayor mortalidad ${ }^{[5][29][30][31][39][41]}$.

Pregunta 4: ¿La escala GRACE predice peor la mortalidad en los pacientes diabéticos?

Para poder contestar esta última pregunta de investigación se ha estudiado el poder predictivo, la calibración y la aplicabilidad clínica del score GRACE en una muestra de 9781 pacientes con SCA, con un 53\% de paciente con SCACEST y un 47\% de pacientes con SCASEST. La prevalencia de DM ha sido aproximadamente del $25 \%$ en el grupo de SCACEST y del 36\% en el grupo de SCASEST.

El poder predictivo del score en nuestra población es adecuada tal y como se objetiva al calcular el área bajo la curva del modelo GRACE para mortalidad hospitalaria, siendo el estadístico C de 0.873 (IC 95\% 0.866 a 0.879). Estos hallazgos confirman el resultado de estudios previos que también han estudiado la validación de la escala GRACE en diferentes poblaciones contemporáneas, obteniendo resultados similares $^{[42][43][44]}$.

La calibración del modelo la calculamos mediante un modelo de regresión lineal entre la mortalidad observada en nuestra población con la mortalidad predicha por el modelo en el estudio original de Granger et al, objetivando muy buena concordancia entre los resultados predichos y observados con un coeficiente de correlación (r) de 0,99. Otros parámetros de calibración calculados apoyan una calibración al menos 
moderada del modelo con un valor para el score de Brier de 0.044 y el estadístico R2 Nagelkerke de $0.34^{[45][46]}$.

Para estudiar la aplicabilidad clínica del modelo, es decir la exactitud diagnóstica del modelo en diferentes subgrupos, hemos calculado el área bajo la curva del modelo aplicado en la población ARIAM, comparando las curvas ROC en función del tipo de SCA (con y sin elevación del ST), y en función de la presencia o no de DM, sin que hayamos encontrado diferencias estadísticamente significativas. Por último hemos querido valorar el posible valor añadido de la variable DM al modelo mediante la Mejora Neta de Reclasificación (NRI) ${ }^{[47]}$. Según los resultados, la variable DM no mejora la capacidad predictiva del modelo con un NRI 0,02 ( IC 95\% -0,0108 a 0,0706).

Parece que podemos afirmar que la escala GRACE continua teniendo un buen poder predictivo para mortalidad hospitalaria por todas las causas a pesar de los avances médicos y terapéuticos acontecidos desde su generación, y por lo tanto, que su utilización como escala de riesgo isquémico, tal y como recomiendan las guías, es adecuado, tanto en la población española actual como en el subgrupo de pacientes diabéticos. 


\section{DISCUSIÓN GENERAL:}

Tras discutir cada uno de los resultados obtenidos, y dado que ambos trabajos surgen del análisis de datos extraídos del mismo registro observacional ambispectivo ARIAM-SEMICYUC, vamos a realizar una serie de consideraciones generales.

El registro ARIAM-SEMICYUC es un registro multicéntrico en el que participan centros de toda España con lo que se obtiene una muestra bien representativa de la población española. Se trata de un registro de base hospitalaria, no de base poblacional, ya que solo se incluyen los pacientes que ingresan en las unidades coronarias que voluntariamente participan en el proyecto, lo cual podría producir un sesgo de selección, al perder aquellos pacientes que ingresan en planta o que fallecen antes de llegar al hospital.

Otra de las limitaciones más importantes del registro es el seguimiento a partir del alta hospitalaria. Para los análisis de mortalidad es preferible utilizar la variable de mortalidad hospitalaria debido al elevado número de pérdidas en la variable de mortalidad a los 30 días. Esta limitación, sin embargo, no tiene porqué haber afectado nuestros resultados puesto que las pérdidas son aleatorias, repartidas igualmente en los diferentes grupos de estudio.

Como ya se ha comentado, los resultados analizados surgen de estudios de cohortes retrospectivos, y su principal problema es la vulnerabilidad al efecto de posibles factores de confusión, por lo que dichos resultados se deben de tomar con precaución.

Tras la publicación de los últimos resultados, referentes a la accesibilidad al sistema sanitario de los pacientes diabéticos con SCACEST, se envían a la revista "Medicina Intensiva" 2 cartas al editor, de las cuales derivamos las respectivas réplicas añadidas a este trabajo. Las consideraciones realizas en estas 2 cartas son similares y no están realmente en relación con el objetivo principal del estudio, si no en relación con las posibles causas del retraso de los pacientes diabéticos con SCA, lo cual ya se ha discutido previamente. 


\section{CONCLUSIÓN FINAL:}

Los pacientes diabéticos siguen presentado una mayor tasa de mortalidad por todas las causas, así como una mayor tasa de complicaciones y eventos cardiovasculares que los pacientes no diabéticos ${ }^{[17][18][19]}$.

Como venimos diciendo desde el principio en este trabajo, son muchas las incertidumbres entorno a la peor evolución de los pacientes diabéticos con SCA. Nosotros hemos podido contestar algunas de ellas.

En primer lugar, los resultados de este trabajo sugieren que los pacientes diabéticos con SCA se manejan de forma mas conservadora, lo cual es consistente con la literatura existente. Las recomendaciones de las guías clínicas son claras y están basadas en un alto nivel de evidencia, y aunque no conozcamos la causa, también parece claro que los clínicos no siguen estas recomendaciones.

En el caso de los pacientes con SCASEST, parece que los pacientes diabéticos tratados de forma conservadora, son un subgrupo caracterizado por su mayor edad y comorbilidad cardiovascular, mayor riesgo hemorrágico y ausencia de ECG de alto riesgo. El temor a las complicaciones hemorrágicas o la presencia de lesiones coronarias avanzadas podrían ser la causa de la infrautilización de la estrategia invasiva en los pacientes diabéticos con SCASEST. Y este hecho podría estar favoreciendo el peor pronóstico de estos pacientes frente a los no diabéticos.

Por otro lado, no parece que existan problemas de accesibilidad al sistema sanitario de los pacientes diabéticos, aunque parece que tardan más en contactar con el sistema, sin que conozcamos bien las causas que motivan este mayor retraso. Sin embargo, lo que parece que determina su mayor mortalidad no es este retraso, si no el hecho de padecer diabetes y la mayor gravedad que presentan al ingreso hospitalario.

Por último, el uso rutinario de la escala de riesgo isquémico GRACE, tal y como recomiendan las guías clínicas, parece adecuado, ya que presenta un buen poder predictivo para mortalidad hospitalaria en la población española actual, sin encontrar diferencias significativas ni en función del tipo de SCA ni en función de la presencia o no de DM. 


\section{BIBLIOGRAFÍA:}

1. Informe de la salud cardiovascular en España en el contexto europeo. 2006.

2. Informe sobre la incidencia cardiovascular en España. 2009.

3. De Miguel Balsa E, Latour Perez J, Fuset Cabanes MP, Ochagavía Calvo A. ARIAM: Análisis del corte 2015. Madrid: 2015.

4. Donahoe SM, Stewart GC, McCabe CH, Mohanavelu S, Murphy SA, Cannon $\mathrm{CP}$, et al. Diabetes and mortality following acute coronary syndromes. JAMA 2007;298(7):765-75.

5. Hasin T, Hochadel M, Gitt AK, Behar S, Bueno H, Hasin Y. Comparison of treatment and outcome of acute coronary syndrome in patients with versus patients without diabetes mellitus. Am J Cardiol 2009;103(6):772-8.

6. Dotevall A, Hasdai D, Wallentin L, Battler A, Rosengren A. Diabetes mellitus: clinical presentation and outcome in men and women with acute coronary syndromes. Data from the Euro Heart Survey ACS. Diabet Med 2005;22(11):1542-50.

7. Roffi M, Patrono C, Collet J, Mueller C, Valgimigli M, Andreotti F, et al. 2015 ESC Guidelines for the management of acute coronary syndromes in patients presenting without persistent ST-segment elevation: Task Force for the Management of Acute Coronary Syndromes in Patients Presenting without Persistent ST-Segment Elevation of . Eur Hear J 2016;37(3):267-315.

8. Amsterdam EA, Wenger NK, Brindis RG, Casey DE, Ganiats TG, Holmes DR, et al. 2014 AHA/ACC guideline for the management of patients with non-STelevation acute coronary syndromes: a report of the American College of Cardiology/American Heart Association Task Force on Practice Guidelines. Circulation 2014;130(25):344-426.

9. Task Force on the management of ST-segment elevation acute myocardial infarction of the European Society of Cardiology (ESC), Steg PG, James SK, Atar D, Badano LP, Blömstrom-Lundqvist C, et al. ESC Guidelines for the management of acute myocardial infarction in patients presenting with STsegment elevation. Eur Heart J 2012;33(20):2569-619. 
10. Windecker S, Kolh P, Alfonso F, Collet J-P, Cremer J, Falk V, et al. 2014 ESC/EACTS Guidelines on myocardial revascularization: The Task Force on Myocardial Revascularization of the European Society of Cardiology (ESC) and the European Association for Cardio-Thoracic Surgery (EACTS)Developed with the special contribution o. Eur Heart J 2014;35(37):2541-619.

11. Civeira Murillo E, Del Nogal Saez F, Alvarez Ruiz AP, Ferrero Zorita J, Alcantara AG, Aguado GH, et al. [The recommendations regarding non-ST segment elevation acute coronary syndrome have been reviewed. SEMICYUC. Spanish Society for Intensive Medicine, Critical Care and Coronary Units]. Med intensiva 2010;34(1):22-45.

12. Norhammar A, Malmberg K, Rydén L, Tornvall P, Stenestrand U, Wallentin L, et al. Under utilisation of evidence-based treatment partially explains for the unfavourable prognosis in diabetic patients with acute myocardial infarction. Eur Heart J 2003;24(9):838-44.

13. Bhatt DL, Roe MT, Peterson ED, Li Y, Chen AY, Harrington RA, et al. Utilization of early invasive management strategies for high-risk patients with non-ST-segment elevation acute coronary syndromes: results from the CRUSADE Quality Improvement Initiative. JAMA 2004;292(17):2096-104.

14. Anselmino M, Bartnik M, Malmberg K, Rydén L, Euro Heart Survey Investigators. Management of coronary artery disease in patients with and without diabetes mellitus. Acute management reasonable but secondary prevention unacceptably poor: a report from the Euro Heart Survey on Diabetes and the Heart. Eur J Cardiovasc Prev Rehabil 2007;14(1):28-36.

15. Gu K, Cowie CC, Harris MI. Diabetes and decline in heart disease mortality in US adults. JAMA 1999;281(14):1291-7.

16. Fox CS, Coady S, Sorlie PD, Levy D, Meigs JB, D'Agostino RB, et al. Trends in cardiovascular complications of diabetes. JAMA 2004;292(20):2495-9.

17. Mak KH, Moliterno DJ, Granger CB, Miller DP, White HD, Wilcox RG, et al. Influence of diabetes mellitus on clinical outcome in the thrombolytic era of acute myocardial infarction. GUSTO-I Investigators. Global Utilization of Streptokinase and Tissue Plasminogen Activator for Occluded Coronary Arteries. J Am Coll Cardiol 1997;30(1):171-9. 
18. Malmberg K, Yusuf S, Gerstein HC, Brown J, Zhao F, Hunt D, et al. Impact of diabetes on long-term prognosis in patients with unstable angina and non-Q-wave myocardial infarction: results of the OASIS (Organization to Assess Strategies for Ischemic Syndromes) Registry. Circulation 2000;102(9):1014-9.

19. Franklin K, Goldberg RJ, Spencer F, Klein W, Budaj A, Brieger D, et al. Implications of diabetes in patients with acute coronary syndromes. The Global Registry of Acute Coronary Events. Arch Intern Med 2004;164(13):1457-63.

20. Rasoul S, Ottervanger JP, Dambrink J-HE, de Boer M-J, Hoorntje JCA, Gosselink ATM, et al. Are patients with non-ST elevation myocardial infarction undertreated? BMC Cardiovasc Disord 2007;7(5):8.

21. Rydén L, Standl E, Bartnik M, Van den Berghe G, Betteridge J, de Boer M-J, et al. Guidelines on diabetes, pre-diabetes, and cardiovascular diseases: executive summary. The Task Force on Diabetes and Cardiovascular Diseases of the European Society of Cardiology (ESC) and of the European Association for the Study of Diabetes (EASD). Eur Heart J 2007;28(1):88-136.

22. Kuntz RE. Importance of considering atherosclerosis progression when choosing a coronary revascularization strategy: the diabetes-percutaneous transluminal coronary angioplasty dilemma. Circulation 1999;99(7):847-51.

23. Goraya TY, Leibson CL, Palumbo PJ, Weston SA, Killian JM, Pfeifer EA, et al. Coronary atherosclerosis in diabetes mellitus: a population-based autopsy study. J Am Coll Cardiol 2002;40(5):946-53.

24. Fox KAA, Clayton TC, Damman P, Pocock SJ, de Winter RJ, Tijssen JGP, et al. Long-term outcome of a routine versus selective invasive strategy in patients with non-ST-segment elevation acute coronary syndrome a meta-analysis of individual patient data. J Am Coll Cardiol 2010;55(22):2435-45.

25. Roffi M, Eberli FR. Diabetes and acute coronary syndromes. Best Pract Res Clin Endocrinol Metab 2009;23(3):305-16.

26. Wallentin L, Lagerqvist B, Husted S, Kontny F, Ståhle E, Swahn E. Outcome at 1 year after an invasive compared with a non-invasive strategy in unstable coronary-artery disease: the FRISC II invasive randomised trial. FRISC II Investigators. Fast Revascularisation during Instability in Coronary artery disease. Lancet 2000;356:9-16. 
27. Elbarouni B, Ismaeil N, Yan RT, Fox KAA, Connelly KA, Baer C, et al. Temporal changes in the management and outcome of Canadian diabetic patients hospitalized for non-ST-elevation acute coronary syndromes. Am Heart J 2011;162(2):347-55.e1.

28. O'Gara P, Kushner F, Ascheim D, Casey DJ, Chung M, de Lemos J, et al. 2013 ACCF/AHA guideline for the management of ST-elevation myocardial infarction: a report of the American College of Cardiology Foundation/American Heart Association Task Force on Practice Guidelines. J Am Coll Cardiol 2013;61(4):e78-140.

29. Woodfield SL, Lundergan CF, Reiner JS, Greenhouse SW, Thompson MA, Rohrbeck SC, et al. Angiographic findings and outcome in diabetic patients treated with thrombolytic therapy for acute myocardial infarction: the GUSTO-I experience. J Am Coll Cardiol 1996;28(7):1661-9.

30. Ribeiro S, Gaspar A, Rocha S, Nabais S, Azevedo P, Salgado A, et al. Predictors of pre-hospital delay in patients with ST-segment elevation myocardial infarction. Rev Port Cardiol orgão Of da Soc Port Cardiol = Port J Cardiol an Off J Port Soc Cardiol 2010;29(10):1521-32.

31. Goldberg RJ, Spencer FA, Fox KAA, Brieger D, Steg PG, Gurfinkel E, et al. Prehospital Delay in Patients With Acute Coronary Syndromes (from the Global Registry of Acute Coronary Events [GRACE]). Am J Cardiol 2009;103(5):598603.

32. Subherwal S, Bach RG, Chen AY, Gage BF, Rao S V, Newby LK, et al. Baseline risk of major bleeding in non-ST-segment-elevation myocardial infarction: the CRUSADE (Can Rapid risk stratification of Unstable angina patients Suppress ADverse outcomes with Early implementation of the ACC/AHA Guidelines) Bleeding Score. Circulation 2009;119(14):1873-82.

33. Antman EM, Cohen M, Bernink PJ, McCabe CH, Horacek T, Papuchis G, et al. The TIMI risk score for unstable angina/non-ST elevation MI: A method for prognostication and therapeutic decision making. JAMA 2000;284(7):835-42.

34. Morrow DA, Antman EM, Charlesworth A, Cairns R, Murphy SA, de Lemos JA, et al. TIMI risk score for ST-elevation myocardial infarction: A convenient, bedside, clinical score for risk assessment at presentation: An intravenous nPA for treatment of infarcting myocardium early II trial substudy. Circulation 2000;102(17):2031-7. 
35. Granger CB, Goldberg RJ, Dabbous O, Pieper KS, Eagle KA, Cannon CP, et al. Predictors of hospital mortality in the global registry of acute coronary events. Arch Intern Med 2003;163(19):2345-53.

36. McNamee R. Regression modelling and other methods to control confounding. Occup Environ Med 2005;62(7):500-6, 472.

37. Mehran R, Pocock SJ, Nikolsky E, Clayton T, Dangas GD, Kirtane AJ, et al. A risk score to predict bleeding in patients with acute coronary syndromes. J Am Coll Cardiol 2010;55(23):2556-66.

38. Steg PG, Huber K, Andreotti F, Arnesen H, Atar D, Badimon L, et al. Bleeding in acute coronary syndromes and percutaneous coronary interventions: position paper by the Working Group on Thrombosis of the European Society of Cardiology. Eur Heart J 2011;32(15):1854-64.

39. Goldberg RJ, Steg PG, Sadiq I, Granger CB, Jackson EA, Budaj A, et al. Extent of, and factors associated with, delay to hospital presentation in patients with acute coronary disease (the GRACE registry). Am J Cardiol 2002;89(7):791-6.

40. Leslie WS, Urie A, Hooper J, Morrison CE. Delay in calling for help during myocardial infarction: reasons for the delay and subsequent pattern of accessing care. Heart 2000;84(2):137-41.

41. Colmenero-Ruiz M, Reina-Toral A, Jimenez-Quintana M., Aguayo-de-Hoyos E, Ruiz-Bailén M, García-Alcántara A, et al. Variabilidad en el manejo del infarto agudo de miocardio en el registro ARIAM en el año 2002. Med Intensiva 2005;29(8):420-9.

42. Elbarouni B, Goodman SG, Yan RT, Welsh RC, Kornder JM, Deyoung JP, et al. Validation of the Global Registry of Acute Coronary Event (GRACE) risk score for in-hospital mortality in patients with acute coronary syndrome in Canada. Am Heart J 2009;158(3):392-9.

43. Fox KAA, Fitzgerald G, Puymirat E, Huang W, Carruthers K, Simon T, et al. Should patients with acute coronary disease be stratified for management according to their risk? Derivation, external validation and outcomes using the updated GRACE risk score. BMJ Open 2014;4(2):e004425.

44. Shaikh MK, Hanif B, Shaikh K, Khan W, Parkash J. Validation of Grace Risk Score in predicting in-hospital mortality in patients with non ST-elevation myocardial infarction and unstable angina. J Pak Med Assoc 2014;64(7):807-11. 
45. Steyerberg EW, Vickers AJ, Cook NR, Gerds T, Gonen M, Obuchowski N, et al. Assessing the performance of prediction models: a framework for traditional and novel measures. Epidemiology 2010;21(1):128-38.

46. Steyerberg EW, Vergouwe Y. Towards better clinical prediction models: seven steps for development and an ABCD for validation. Eur Heart $J$ 2014;35(29):1925-31.

47. Kerr KF, Wang Z, Janes H, McClelland RL, Psaty BM, Pepe MS. Net reclassification indices for evaluating risk prediction instruments: a critical review. Epidemiology 2014;25(1):114-21. 
- ANDORRA:

Hospital Nostra Senyora de Meritxell

- ANDALUCIA:

Complejo Hospitalario Nuestra Señora de Valme

Complejo Hospitalario Carlos Haya

Hospital Comarcal Santa Ana de Motril

- ARAGON:

Hospital Clínico Universitario Lozano Blesa

Hospital General de la Defensa de Zaragoza

Hospital General San Jorge

Hospital Universitario Miguel Servet

Hospital Maz (Mutua de Accidentes de Zaragoza)

Hospital Nuestra Señora de Gracia

- CANARIAS:

Hospital Quirón Tenerife

- CASTILLA Y LEON:

Complejo Asistencial de Segovia

Complejo Asistencial de Soria

Complejo Asistencial Universitario de Burgos

Hospital El Bierzo

Hospital Universitario del Río Hortega

- CASTILLA LA MANCHA:

Hospital General Universitario de Ciudad Real

Hospital Universitario de Guadalajara

- CATALŨ̃̃a:

Hospital de Sabadell

Hospital General de Catalunya

Hospital de Sant Joan de Déu - Althaia

Hospital de Terrassa

Hospital Universitari Mútua de Terrassa 
- COMUNIDAD VALENCIANA:

Hospital General Universitario de Alicante

Hospital Universitari Sant Joan d'Alacant

Hospital General de Elche

Hospital de La A.V.S. Vega Baja

Hospital de Torrevieja

Hospital Universitario del Vinalopó

Hospital Virgen de los Lirios

Hospital Arnau de Vilanova

Hospital Clínico Universitario

Hospital de Manises

Hospital de Llíria

Hospital Francesc de Borja de Gandía

Hospital Universitari i Politècnic la Fe

Hospital de Sagunto

Hospital de Requena

Hospital Universitario de la Ribera

Hospital Universitario Dr. Peset

Hospital General de Castelló

Hospital de La Plana

- EXTREMADURA:

Complejo Hospitalario de Cáceres

Complejo Hospitalario del Área de Salud de Mérida

Hospital Virgen del Puerto

- GALICIA:

Complejo Hospitalario de Ourense

Complejo Hospitalario Arquitecto Marcide-Prof.

Novoa Santos

Hospital HM Modelo

Complejo Hospitalario Universitario A Coruña

Complejo Hospitalario Xeral-Calde

- ISLAS BALEARES:

Hospital Universitario Son Espases 
- LA RIOJA:

Complejo Hospital San Pedro

- MADRID:

Hospital de Torrejón

Hospital Universitario Príncipe de Asturias

Hospital del Sureste

Hospital del Tajo

Hospital Universitario Severo Ochoa

Hospital Infanta Elena

Hospital Quirón Salud Sur

Hospital Universitario del Henares

Hospital Universitario Fundación Alcorcón

- MURCIA:

Complejo Hospitalario Universitario de Cartagena

Hospital Los Arcos

Hospital Universitario Virgen de la Arrixaca

Hospital General Universitario Reina Sofía

Hospital J.M. Morales Meseguer

- PAIS VASCO:

Hospital Santiago Apóstol

- PRINCIPADO DE ASTURIAS:

Hospital San Agustín 
- Medicina Clínica. Factor de impacto (2015): $1,267$.

La revista "Medicina Clínica", fundada en 1943, es la única publicación semanal de contenido clínico que se edita en España y constituye el máximo exponente de la calidad y pujanza de la medicina española. El principal frente de trabajo de Medicina Clínica son trabajos de investigación original rigurosamente seleccionados atendiendo a su calidad, originalidad e interés.

- Medicina Intensiva. Factor de Impacto (2015): 1,193.

Medicina Intensiva es la revista de la Sociedad Española de Medicina Intensiva, Crítica y Unidades Coronarias y de la Federación Panamericana e Ibérica de Sociedades de Medicina Crítica y Terapia Intensiva. Medicina Intensiva se ha convertido en la publicación de referencia en castellano de la especialidad. Medicina Intensiva publica principalmente artículos Originales, Revisiones, Artículos de consenso, Imágenes en Medicina Intensiva e Información relevante de la especialidad.

\section{- International Journal of Cardiology. Factor de Impacto (2015): 4, 638}

International Journal of Cardiology es una revista que engloba la patología cardiaca, cariometabólica y cardiovascular. Publica artículos de investigación primaria, observacionales y experimentales, así como revisiones bibliográficas siempre que tengan relevancia científica y clínica. La revista cubre todos los aspectos de la cardiología desde los aspectos genéticos hasta poblacionales. 


\section{ANEXO 3: Artículos:}

- Artículo 1: Uso de estrategia invasiva precoz en pacientes diabéticos con sindrome coronario agudo sin elevación del ST.

○ Publicado en "Medicina Clínica".

- Articulo 2: Accesibilidad al sistema sanitario de los pacientes diabéticos con síndrome coronario agudo con elevación del segmento $S T$.

○ Publicado en "Medicina Intensiva".

- Replica de los autores I y II. Publicadas en "Medicina Intensiva", como respuesta a las siguientes cartas:

1. Comentarios al artículo: Accesibilidad al sistema sanitario de los pacientes diabéticos con síndrome coronario agudo con elevación del segmento ST.

2. Educación y concienciación en el paciente diabético con síndrome coronario agudo con elevación del ST.

- Articulo 3: Poder predictivo del score GRACE en la población diabética española.

- Mandado para su consideración a la revista: "International Journal of cardiology", como: "Predictive power of the grace score in the diabetic population”. 


\title{
Original
}

\section{Uso de estrategia invasiva precoz en pacientes diabéticos con síndrome coronario agudo sin elevación del ST}

\author{
Anna Baeza Román ${ }^{\text {a,* }}$, Jaime Latour Pérez ${ }^{a}$, Eva de Miguel Balsa ${ }^{a}$, Karel Pino Izquierdo a , \\ Francisco Javier Coves Orts ${ }^{\mathrm{a}}$, Luis García Ochando ${ }^{\mathrm{b}}$, Maria José de la Torre Fernández ${ }^{\mathrm{c}}$ y \\ Grupo ARIAM-SEMICYUC ${ }^{\diamond}$
}

a Unidad de Cuidados Intensivos, Hospital General Universitario de Elche, Elche, Alicante, España
b Unidad de Cuidados Intensivos, Hospital Dr. Peset, Valencia, España

${ }^{c}$ Unidad de Cuidados Intensivos, Complexo Hospitalario Universitario de Ourense, Ourense, España

\section{IN F ORMACIÓN DEL ARTÍCULO}

\section{Historia del artículo:}

Recibido el 18 de noviembre de 2012

Aceptado el 31 de enero de 2013

On-line el 17 de abril de 2013

\section{Palabras clave:}

Síndrome coronario agudo sin elevación de ST

Estrategia invasiva precoz

Diabetes mellitus

\begin{abstract}
R E S U M E N
Fundamento y objetivos: La estrategia invasiva reduce la mortalidad de los pacientes con síndrome coronario agudo sin elevación del ST (SCASEST) de alto riesgo, entre los que se incluyen los pacientes diabéticos. Sin embargo, diversas publicaciones han puesto de manifiesto una infrautilización de esta estrategia invasiva en estos pacientes. El objetivo de este estudio es conocer las características de los pacientes diabéticos que se tratan de forma conservadora, e identificar determinantes del uso de una u otra estrategia.

Paciente y métodos: Estudio de cohortes retrospectivo, realizado en diabéticos con SCASEST incluidos en los cortes anuales de 2010 y 2011 en el registro ARIAM-SEMICYUC. Se realizó un análisis bruto y ajustado mediante regresión logística no condicional.

Resultados: Se han analizado 531 pacientes diabéticos, de los cuales $264(49,7 \%)$ recibieron estrategia invasiva. Los pacientes diabéticos que se tratan de forma conservadora son un subgrupo caracterizado por su mayor edad y comorbilidad cardiovascular, mayor riesgo hemorrágico y ausencia de electrocardiograma (ECG) de alto riesgo. Se identifican como variables predictoras independientes asociadas al tratamiento conservador, el ECG de bajo riesgo, el Killip al ingreso superior a 1, alto riesgo hemorrágico y el tratamiento previo con clopidogrel.

Conclusiones: El temor a las complicaciones hemorrágicas o la presencia de lesiones coronarias avanzadas podrían ser la causa de la infrautilización de la estrategia invasiva en los pacientes diabéticos con SCASEST.
\end{abstract}

() 2012 Elsevier España, S.L. Todos los derechos reservados.
Keywords:

Acute coronary syndrome without ST elevation

Early invasive strategy

Diabetes mellitus

\section{Early invasive strategy in diabetic patients with non-ST-segment elevation acute coronary syndromes}

\section{A B S T R A C T}

Background and objectives: In the management of non-ST-segment elevation acute coronary syndromes (NSTE-ACS), several studies have shown a reduction in mortality with the use of an invasive strategy in high-risk patients, including diabetic patients. Paradoxically, other studies have shown an underutilization of this invasive strategy in these patients. The aim of this study is to determine the characteristics of patients managed conservatively and identify determinants of the use of invasive or conservative strategy.

Patients and Methods: Retrospective cohort study conducted in diabetic patients with NSTE-ACS included in the ARIAM-SEMICYUC registry $(n=531)$ in 2010 and 2011. We performed crude and adjusted unconditional logistic regression.

\footnotetext{
* Autor para correspondencia.

Correo electrónico: anna.baeza@coma.es (A. Baeza Román).

$\diamond$ Más información sobre el Grupo ARIAM-SEMICYUC está disponible en el Anexo.
} 
Results: We analyzed 531 diabetic patients, 264 (49.7\%) of which received invasive strategy. Patients managed conservatively were a subgroup characterized by older age and cardiovascular comorbidity, increased risk of bleeding and the absence of high-risk electrocardiogram (ECG). In diabetic patients with NSTE-ACS, independent predictors associated with conservative strategy were low-risk ECG, initial Killip class $>1$, high risk of bleeding and pretreatment with clopidogrel.

Conclusions: The fear of bleeding complications or advanced coronary lesions could be the cause of the underutilization of an invasive strategy in diabetic patients with NSTE-ACS.

(c) 2012 Elsevier España, S.L. All rights reserved.

\section{Introducción}

Aproximadamente un $20-30 \%$ de los pacientes con síndrome coronario agudo sin elevación de ST (SCASEST) tiene diabetes conocida, y al menos la misma proporción tiene diabetes no diagnosticada o intolerancia a la glucosa.

La diabetes mellitus (DM) es un predictor independiente de mortalidad e infarto de miocardio (IM) no fatal ${ }^{1-4}$. Diversos estudios han demostrado una reducción de la mortalidad con el uso de la estrategia invasiva en enfermos de alto riesgo, entre los que se incluyen los pacientes diabéticos ${ }^{5-9}$. Por este motivo, las principales guías clínicas del manejo del SCASEST recomiendan el uso de la estrategia invasiva en estos pacientes ${ }^{10-12}$.

Norhammar et al. encontraron en su estudio una infrautilización del tratamiento, basado en la evidencia, tanto farmacológico como de revascularización en los pacientes diabéticos ${ }^{13}$. Demostraron, además, que el tratamiento conseguía el mismo beneficio en ambos grupos de pacientes, pudiendo ser la infrautilización del tratamiento en los diabéticos la causa del incremento de mortalidad y de efectos adversos en este grupo. En un estudio posterior, Norhammar et al. sugirieron que el tratamiento invasivo precoz en los pacientes diabéticos tiene mayor beneficio que en los no diabéticos, con un número necesario a tratar (NNT) para evitar IM o muerte de 11 , frente a un NNT de 32 para pacientes no diabéticos ${ }^{14}$. Hallazgos similares se encontraron en otros estu$\operatorname{dios}^{2,8,15-17}$. Finalmente, Henriksson et al. mostraron en su estudio que la estrategia invasiva es coste-efectiva en estos pacientes ${ }^{18}$.

Sin embargo, no conocemos bien el perfil específico de los pacientes diabéticos que se tratan de forma conservadora. El objetivo de este estudio es identificar determinantes del uso de una $\mathrm{u}$ otra estrategia.

\section{Pacientes y método}

Se trata de un estudio observacional retrospectivo basado en los datos del Registro ARIAM-SEMICYUC en el que participan diferentes hospitales españoles ( 43 centros en 2010 y 56 centros en 2011). Se han recogido los datos de aquellos pacientes consecutivos que ingresan en la Unidad de Cuidados Intensivos con diagnóstico al ingreso de SCASEST y que además presentan DM.

La estrategia invasiva precoz se define como la realización de angiografía coronaria seguida o no de revascularización, tanto de forma urgente como programada, en las primeras $24 \mathrm{~h}$ de ingreso. El resto de los pacientes se incluyen en el grupo de tratamiento conservador.

Las variables analizadas incluyen factores sociodemográficos (edad y sexo), factores de riesgo cardiovascular (hipertensión arterial, dislipidemia, tabaquismo y obesidad), antecedentes personales (arteriopatía periférica, insuficiencia cardiaca, IM, angina, lesiones coronarias conocidas, accidente cerebrovascular, enfermedad pulmonar obstructiva crónica e insuficiencia renal crónica), tratamiento médico previo (ácido acetil salicílico, clopidogrel, acenocumarol, inhibidores de la enzima conversiva de la angiotensina, antagonistas de los receptores de la angiotensina II, betabloqueantes, estatinas, digoxina y otros antiarrítmicos), forma clínica de presentación (presencia de electrocardiograma [ECG] de alto riesgo, arritmias, grado de insuficiencia cardiaca y presencia de troponinas positivas), presencia de centro con disponibilidad de laboratorio de hemodinámica, puntuaciones de riesgo isquémico basal (GRACE y TIMI), y el riesgo hemorrágico codificado en bajo, medio y alto según la puntuación del estudio CRUSADE ${ }^{19}$. El ECG de riesgo se define como cambios evidentes de afectación de tronco de coronaria izquierda, el descenso de ST de más de $2 \mathrm{~mm}$ en más de 2 derivaciones, la elevación transitoria de ST, la fibrilación ventricular primaria y el bloqueo auriculoventricular de alto grado al ingreso ${ }^{20}$.

Los datos han sido analizados con SPSS ${ }^{\circledR}$ Statistics para Windows versión 19.0. En la estadística descriptiva las variables cuantitativas continuas se expresan como la mediana y percentiles 25 y 75, y las variables categóricas, como proporciones. La comparación de variables categóricas se realiza con el test ji al cuadrado y la comparación de las variables cuantitativas mediante el test de Mann-Whitney tras comprobar que dichas variables no siguen una distribución normal mediante la prueba de ShapiroWilks. El análisis de contraste se realiza de forma bilateral con un nivel de significación $\alpha$ del 5\%. Finalmente, se realiza una regresión logística binaria para determinar las variables predictoras independientes asociadas al uso de la estrategia invasiva precoz (EIP). Se incluyen en el modelo todas aquellas variables que en el análisis univariante habían obtenido un nivel de significación $\mathrm{p} \leq 0,1$, y se realiza mediante estrategia hacia atrás.

La asociación entre las variables predictoras y la realización de una EIP se estima mediante el cálculo de las odds ratio ajustadas y de sus intervalos de confianza del $95 \%$.

Por último, dado que algunas de las guías clínicas recomiendan, en pacientes diabéticos de bajo riesgo, la realización del cateterismo dentro de las primeras $72 \mathrm{~h}^{11}$, se realizó un análisis similar, pero incluyendo en el grupo de estrategia invasiva aquellos pacientes sometidos a coronariografía dentro de este período de tiempo.

\section{Resultados}

El período de reclutamiento del registro ARIAM-SEMICYUC ha sido del 1 de abril al 15 de julio del año 2010 y del 15 de abril al 14 de julio de 2011. De un total de 3.238 pacientes con SCA incluidos en el registro, 1.943 tenían el diagnóstico de SCASEST al ingreso. El $27,3 \%$ de estos pacientes padecían además DM. En total, se han analizado 531 pacientes diabéticos con SCASEST, de los cuales 264 $(49,7 \%)$ recibieron estrategia invasiva.

La tabla 1 muestra las características clínicas de los 2 grupos. No se encontraron diferencias significativas en el sexo ni en los factores de riesgo cardiovascular; sin embargo, los pacientes que se trataron con estrategia invasiva fueron más jóvenes. Respecto a la comorbilidad y el tratamiento previo, los pacientes que recibieron estrategia invasiva tenían menos antecedentes de insuficiencia cardiaca y de IM previo. Tenían, además, menor prevalencia de lesiones coronarias conocidas y recibieron con menos frecuencia tratamiento previo con clopidogrel. Respecto a la forma de presentación clínica, tenían al ingreso mayor proporción de ECG de riesgo y menor proporción de pacientes con grupo de Killip mayor de 1. El riesgo isquémico fue menor en el grupo de EIP, y con menos frecuencia presentaban un riesgo hemorrágico alto. 
Tabla 1

Características basales de los pacientes según la estrategia utilizada

\begin{tabular}{|c|c|c|c|}
\hline \multirow[t]{2}{*}{ Características basales } & \multicolumn{2}{|c|}{ Estrategia invasiva precoz } & \multirow[t]{2}{*}{$\mathrm{p}$} \\
\hline & No $(n=267)$ & Sí $(n=264)$ & \\
\hline Edad, mediana ( $p 25-p 75)$ & $74(64-80)$ & $72(62-77)$ & 0,025 \\
\hline Sexo (varones) & $172(64,4 \%)$ & $185(70,1 \%)$ & 0,167 \\
\hline \multicolumn{4}{|l|}{ Factores de riesgo cardiovascular } \\
\hline Hipertensión arterial & $207(77,5 \%)$ & $204(77,3 \%)$ & 0,944 \\
\hline Dislipidemia & $171(64 \%)$ & $166(62,9 \%)$ & 0,788 \\
\hline Tabaquismo & $106(40 \%)$ & $120(45,8 \%)$ & 0,188 \\
\hline Obesidad & $102(38,2 \%)$ & $98(37,1 \%)$ & 0,858 \\
\hline \multicolumn{4}{|l|}{ Antecedentes personales } \\
\hline Arteriopatía periférica & $42(15,7 \%)$ & $27(10,2 \%)$ & 0,071 \\
\hline Insuficiencia cardiaca & $32(12 \%)$ & $14(5,3 \%)$ & 0,008 \\
\hline Infarto de miocardio & $91(34,1 \%)$ & $60(22,7 \%)$ & 0,004 \\
\hline Angina & $58(21,7 \%)$ & $62(23,5 \%)$ & 0,678 \\
\hline Lesiones coronarias conocidas & $91(34,1 \%)$ & $67(25,4 \%)$ & 0,029 \\
\hline Accidente cerebrovascular & $15(5,6 \%)$ & $18(6,8 \%)$ & 0,594 \\
\hline Enfermedad pulmonar obstructiva crónica & $32(12 \%)$ & $33(12,5 \%)$ & 0,895 \\
\hline Insuficiencia renal crónica & $33(12,4 \%)$ & $23(8,7 \%)$ & 0,204 \\
\hline \multicolumn{4}{|l|}{ Tratamiento médico previo } \\
\hline AAS & $131(49,1 \%)$ & $115(43,6 \%)$ & 0,223 \\
\hline Clopidogrel & $66(24,7 \%)$ & $40(15,2 \%)$ & 0,007 \\
\hline Acenocumarol & $36(13,5 \%)$ & $22(8,3 \%)$ & 0,07 \\
\hline IECA y ARA-II & $130(48,7 \%)$ & $123(46,6 \%)$ & 0,664 \\
\hline Betabloqueantes & $83(31,1 \%)$ & $75(28,4 \%)$ & 0,508 \\
\hline Estatinas & $155(58,1 \%)$ & $135(51,1 \%)$ & 0,117 \\
\hline Digoxina & $5(1,9 \%)$ & $7(2,7 \%)$ & 0,575 \\
\hline Otros antiarrítmicos & $6(2,2 \%)$ & $8(3 \%)$ & 0,601 \\
\hline \multicolumn{4}{|l|}{ Forma clínica de presentación } \\
\hline ECG riesgo & $83(31,1 \%)$ & $106(40,2 \%)$ & 0,03 \\
\hline Arritmias & $62(24,8 \%)$ & $53(20,9 \%)$ & 0,34 \\
\hline Killip > 1 & $128(47,9 \%)$ & $80(30,3 \%)$ & $<0,001$ \\
\hline Troponina positiva & $223(87,8 \%)$ & $214(89,5 \%)$ & 0,572 \\
\hline Disponibilidad de hemodinámica & $63(23,6 \%)$ & $82(31,1 \%)$ & 0,064 \\
\hline \multicolumn{4}{|l|}{ Puntuaciones de riesgo isquémico basal } \\
\hline GRACE, mediana (p25-p75) & $142,9(115-169)$ & $134,6(108-158,5)$ & 0,024 \\
\hline $\mathrm{TIMI}>4$ & $65(24,3 \%)$ & $50(18,9 \%)$ & 0,141 \\
\hline Riesgo hemorrágico alto (puntuación CRUSADE) & $222(83,1 \%)$ & $189(71,6 \%)$ & 0,002 \\
\hline
\end{tabular}

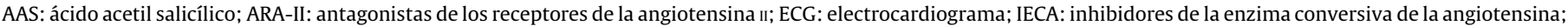
p25-p75: percentil 25-percentil 75

*La estrategia invasiva precoz incluye las primeras $24 \mathrm{~h}$ de ingreso.

De todas estas variables, el modelo de regresión logística binaria identificó 4 variables independientes predictoras asociadas al uso de tratamiento invasivo (tabla 2): 1) presencia de ECG de riesgo; 2 ) ausencia de tratamiento previo con clopidogrel; 3) ausencia al ingreso de Killip > 1, y 4) ausencia de riesgo hemorrágico alto.

Con el objeto de descartar un sesgo de selección, se realizó un análisis de sensibilidad consistente en la exclusión del estudio de los pacientes que fallecieron en las primeras $24 \mathrm{~h}$ (18 pacientes, $3,4 \%$ ). Tras excluir las muertes precoces, el análisis multivariante tuvo resultados similares. Estos resultados se muestran en la tabla 3.

En un segundo análisis se incluyeron en el grupo de estrategia invasiva aquellos pacientes sometidos a coronariografía en las

Tabla 2

Variables predictoras independientes asociadas al uso de estrategia invasiva precoz

\begin{tabular}{lrr}
\hline Variables predictoras & OR (IC 95\%) & \multicolumn{1}{c}{$\mathrm{p}$} \\
\hline ECG de riesgo & $1,69(1,153-2,476)$ & 0,007 \\
Clopidogrel & $0,645(0,407-1,023)$ & 0,062 \\
Killip $>1$ & $0,499(0,341-0,732)$ & $<0,001$ \\
Riesgo hemorrágico alto & $0,666(0,425-1,044)$ & 0,076 \\
\hline
\end{tabular}

ECG: electrocardiograma; IC 95\%: intervalo de confianza del 95\%; OR: odds ratio. primeras $72 \mathrm{~h}$. Los pacientes tratados de forma invasiva tuvieron características muy similares a los del grupo de EIP del análisis previo. La única diferencia es que con menos frecuencia presentaban tratamiento previo con acenocumarol, y no se encontraron diferencias significativas respecto a la presencia de lesiones coronarias significativas (tabla 4). En este caso, el modelo de regresión logística identificó las mismas variables independientes predictoras asociadas al uso de tratamiento invasivo, añadiendo una quinta variable: la ausencia de tratamiento previo con acenocumarol (tabla 5). Además, estos resultados no varían al excluir del análisis a los pacientes fallecidos en las primeras $24 \mathrm{~h}$ (19 pacientes, 3,6\%). Estos resultados se muestran en la tabla 6.

Tabla 3

Variables predictoras independientes asociadas al uso de estrategia invasiva precoz tras excluir del análisis a los pacientes fallecidos en las primeras $24 \mathrm{~h}$

\begin{tabular}{lll}
\hline Variables predictoras & OR (IC 95\%) & $\mathrm{p}$ \\
\hline ECG de riesgo & $1,794(1,214-2,649)$ & 0,003 \\
Clopidogrel & $0,644(0,402-1,031)$ & 0,067 \\
Killip $>1$ & $0,509(0,344-0,754)$ & 0,001 \\
Riesgo hemorrágico alto & $0,685(0,436-1,075)$ & 0,1 \\
\hline
\end{tabular}

ECG: electrocardiograma; IC 95\%: intervalo de confianza del 95\%; OR: odds ratio. 
Tabla 4

Características basales de los pacientes según la estrategia utilizada

\begin{tabular}{|c|c|c|c|}
\hline \multirow[t]{2}{*}{ Características basales } & \multicolumn{2}{|c|}{ Estrategia invasiva } & \multirow[t]{2}{*}{$\mathrm{p}$} \\
\hline & No $(n=192)$ & Sí $(\mathrm{n}=339)^{\mathrm{a}}$ & \\
\hline Edad, mediana ( $p 25-p 75)$ & $75(65-80)$ & $71(62-77)$ & 0,002 \\
\hline Sexo (varones) & $122(63,5 \%)$ & $235(69,3 \%)$ & 0,179 \\
\hline \multicolumn{4}{|l|}{ Factores de riesgo cardiovascular } \\
\hline Hipertensión arterial & $156(81,3 \%)$ & $255(75,2 \%)$ & 0,13 \\
\hline Dislipidemia & $127(66,1 \%)$ & $215(61,9 \%)$ & 0,35 \\
\hline Tabaquismo & $76(39,8 \%)$ & $150(44,6 \%)$ & 0,314 \\
\hline Obesidad & $75(39,1 \%)$ & $125(36,9 \%)$ & 0,642 \\
\hline \multicolumn{4}{|l|}{ Antecedentes personales } \\
\hline Arteriopatía periférica & $31(16,1 \%)$ & $38(11,2 \%)$ & 0,109 \\
\hline Insuficiencia cardiaca & $26(13,5 \%)$ & $20(5,9 \%)$ & 0,004 \\
\hline Infarto de miocardio & $66(34,4 \%)$ & $85(25,1 \%)$ & 0,027 \\
\hline Angina & $43(22,4 \%)$ & $77(22,7 \%)$ & 0,99 \\
\hline Lesiones coronarias conocidas & $66(34,4 \%)$ & $92(27,1 \%)$ & 0,093 \\
\hline Accidente cerebrovascular & $8(4,2 \%)$ & $25(7,4 \%)$ & 0,19 \\
\hline Enfermedad pulmonar obstructiva crónica & $25(13 \%)$ & $40(11,8 \%)$ & 0,681 \\
\hline Insuficiencia renal crónica & $27(14,1 \%)$ & $29(8,6 \%)$ & 0,056 \\
\hline \multicolumn{4}{|l|}{ Tratamiento médico previo } \\
\hline AAS & $92(47,9 \%)$ & $154(45,4 \%)$ & 0,588 \\
\hline Clopidogrel & $50(26 \%)$ & $56(16,5 \%)$ & 0,009 \\
\hline Acenocumarol & $32(16,7 \%)$ & $26(7,7 \%)$ & 0,002 \\
\hline IECA y ARA-II & $99(51,6 \%)$ & $154(45,4 \%)$ & 0,177 \\
\hline Betabloqueantes & $61(31,8 \%)$ & $97(28,6 \%)$ & 0,489 \\
\hline Estatinas & $114(59,4 \%)$ & $176(51,9 \%)$ & 0,103 \\
\hline Digoxina & $5(2,6 \%)$ & $7(2,1 \%)$ & 0,764 \\
\hline Otros antiarrítmicos & $6(3,1 \%)$ & $8(2,4 \%)$ & 0,585 \\
\hline \multicolumn{4}{|l|}{ Forma clínica de presentación } \\
\hline ECG riesgo & $55(28,6 \%)$ & $134(39,5 \%)$ & 0,014 \\
\hline Arritmias & $45(25,4 \%)$ & $70(21,5 \%)$ & 0,319 \\
\hline Killip $>1$ & $95(49,5 \%)$ & $113(33,3 \%)$ & $<0,001$ \\
\hline Troponina positiva & $161(87 \%)$ & $276(89,6 \%)$ & 0,383 \\
\hline Disponibilidad de hemodinámica & $46(24 \%)$ & $99(29,2 \%)$ & 0,224 \\
\hline \multicolumn{4}{|l|}{ Puntuaciones de riesgo isquémico basal } \\
\hline GRACE, mediana (p25-p75) & $143(115-172)$ & $135(108-160)$ & 0,024 \\
\hline TIMI $>4$ & $47(24,5 \%)$ & $68(20,1 \%)$ & 0,273 \\
\hline Riesgo hemorrágico alto (puntuación CRUSADE) & $164(85,4 \%)$ & $247(72,9 \%)$ & 0,001 \\
\hline
\end{tabular}

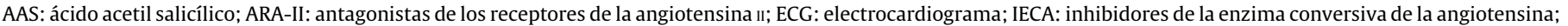
p25-p75: percentil 25-percentil 75.

${ }^{\text {a }}$ La estrategia invasiva incluye las primeras $72 \mathrm{~h}$ de ingreso.

\section{Discusión}

Los resultados del estudio evidencian que los pacientes diabéticos que se tratan de forma conservadora son un subgrupo caracterizado por su mayor edad y comorbilidad cardiovascular, mayor riesgo hemorrágico y ausencia de ECG de alto riesgo.

El hecho de que los pacientes sin cambios importantes en el ECG y con riesgo hemorrágico alto se traten de forma conservadora responde a la recomendación de las guías clínicas. Sin embargo, estas mismas guías recomiendan el uso de una estrategia invasiva en los pacientes de más gravedad y con mayor grado de insuficiencia cardiaca ${ }^{10-12}$, lo cual no se ve reflejado en los resultados de nuestro estudio.

Tabla 5

Variables predictoras independientes asociadas al uso de la estrategia invasiva

\begin{tabular}{lll}
\hline Variables predictoras & OR (IC 95\%) & $\mathrm{p}$ \\
\hline ECG de riesgo & $1.770(1.178-2.660)$ & 0,006 \\
Clopidogrel & $0,606(0,382-0,959)$ & 0,033 \\
Acenocumarol & $0,498(0,279-0,888)$ & 0,018 \\
Killip $>1$ & $0,581(0,393-0,860)$ & 0,007 \\
Riesgo hemorrágico alto & $0,607(0,369-0,999)$ & 0,050 \\
\hline
\end{tabular}

ECG: electrocardiograma; IC 95\%: intervalo de confianza del 95\%; OR: odds ratio.
En el grupo tratado de forma conservadora existe mayor prevalencia de lesiones conocidas e infarto previo, por lo que es posible que las lesiones coronarias en este grupo fueran de mayor gravedad (enfermedad multivaso, no revascularizables, etc.) y condicionasen la decisión del tratamiento conservador. En el registro ARIAM-SEMICYUC se recoge el antecedente de lesiones coronarias conocidas como existencia de lesiones coronarias significativas (>50\%) demostradas mediante coronariografía u otras técnicas de imagen, pero no se recoge si dichas lesiones son no revascularizables. Elbarouni et al. también muestran una infrautilización de las maniobras de revascularización en los pacientes diabéticos con SCASEST, y al analizar los posibles motivos, la principal causa encontrada fue la existencia de

Tabla 6

Variables predictoras independientes asociadas al uso de estrategia invasiva tras excluir del análisis a los pacientes fallecidos en las primeras $24 \mathrm{~h}$

\begin{tabular}{lll}
\hline Variables predictoras & OR (IC 95\%) & $\mathrm{p}$ \\
\hline ECG de riesgo & $1.934(1.268-2.952)$ & 0,002 \\
Clopidogrel & $0,593(0,371-0,958)$ & 0,032 \\
Acenocumarol & $0,483(0,269-0,867)$ & 0,015 \\
Killip $>1$ & $0,627(0,419-0,939)$ & 0,023 \\
Riesgo hemorrágico alto & $0,644(0,390-1.063)$ & 0,085 \\
\hline
\end{tabular}

ECG: electrocardiograma; IC 95\%: intervalo de confianza del 95\%; OR: odds ratio. 
lesiones conocidas no revascularizables o el rechazo del paciente o la familia al procedimiento ${ }^{9}$.

El motivo de que los pacientes con mayor grado de insuficiencia cardiaca congestiva (ICC) se traten de forma conservadora no está claro. En nuestro estudio, los pacientes que se han tratado de forma conservadora presentaban ICC con Killip $>1$, pero además tenían antecedentes de ICC e IM previos, lo cual podría estar en relación con enfermedad coronaria avanzada.

Habitualmente, en los hospitales que no disponen de laboratorio de hemodinámica, la realización de un cateterismo de forma precoz supone un problema. En nuestro estudio, la EIP se ha realizado un 10,4\% más en hospitales con hemodinámica; sin embargo, esta diferencia no ha resultado estadísticamente significativa en el análisis univariante. Nuestro estudio no tiene potencia estadística suficiente para detectar una diferencia como esta, lo que no significa que no exista.

Las recomendaciones de las guías clínicas son claras y están basadas en un alto nivel de evidencia. Y aunque no conozcamos la causa, también parece claro que los clínicos no siguen estas recomendaciones. El temor a las complicaciones hemorrágicas o la presencia de lesiones coronarias avanzadas podrían ser las causas de la infrautilización de la estrategia invasiva en los pacientes diabéticos con SCASEST. Y este hecho podría estar favoreciendo el peor pronóstico de los pacientes diabéticos con SCASEST frente a los no diabéticos.

\section{Conflicto de intereses}

Los autores declaran no tener ningún conflicto de intereses.

\section{Anexo}

El Grupo ARIAM-SEMICYUC está formado por varios hospitales de España y Andorra.

\section{Bibliografía}

1. Donahoe SM, Stewart GC, McCabe CH, Mohanavelu S, Murphy SA, Cannon CP, et al. Diabetes and mortality following acute coronary syndromes. JAMA 2007;298:765-75

2. Hasin T, Hochadel M, Gitt A, Behar S, Bueno H, Hasin Y. Comparison of treatment and outcome of acute coronary syndrome in patients with versus patients without diabetes mellitus. Am J Cardiol. 2009;103:772-8.

3. Quirós R, García J, Martín MD, Trujillo J, Villena MA, Perea E. Factores pronósticos y de supervivencia a largo plazo tras el diagnóstico inicial de insuficiencia cardiaca. Med Clin (Barc). 2012;138:602-8

4. García C, Lupón J, Urrutia A, González B, Herreros J, Altimir S, et al. Significado pronóstico de la diabetes mellitus en una población con insuficiencia cardíaca: mortalidad e ingreso por insuficiencia cardíaca al cabo de un año. Med Clin (Barc). 2005;125:161-5.

5. Fox KA, Clayton TC, Damman P, Pocock SJ, de Winter RJ, Tijssen JG, et al.; FIR Collaboration. Long-term outcome of a routine versus selective invasive strategy in patients with non-ST-segment elevation acute coronary syndrome a meta-analysis of individual patient data. J Am Coll Cardiol. 2010;55: 2435-45.

6. Roffi M, Eberli F. Diabetes and acute coronary syndromes. Best Pract Res Clin Endocrinol Metab. 2009;23:305-16.

7. Wallentin L, Lagerqvist B, Husted S, Ståhle E, Swahn E. Outcome at 1 year after an invasive compared with a non-invasive strategy in unstable coronary-artery disease: The FRISC II invasive randomised trial. Lancet. 2000;356:9-16.

8. Bhatt DL, Roe MT, Peterson ED, Li Y, Chen AY, Harrington RA, et al. Utilization of early invasive management strategies for high-risk patients with non-ST-segment elevation acute coronary syndromes: Results from the CRUSADE quality improvement initiative. JAMA. 2004;292:2096-104.

9. Elbarouni B, Ismaeil N, Yan R, Fox KA, Connelly KA. Baer C, et al. Temporal changes in the management and outcome of Canadian diabetic patients hospitalized for non-ST-elevation acute coronary syndromes. Am Heart J. 2011; 162:347-55.

10. 2012 Writing Committee Members, Jneid H, Anderson JL, Wright RS, Adams CD, Bridges CR, Casey DE, et al. 2012 ACCF/AHA focused update of the guideline for the management of patients with unstable angina/non-ST-elevation myocardial infarction (updating the 2007 guideline and replacing the 2011 focused update): A report of the American College of Cardiology Foundation/American Heart Association Task Force on practice guidelines. Circulation. 2012;126: 875-910.

11. Hamm CW, Bassand JP, Agewall S, Bax J, Boersma E, Bueno H, et al. ESC guidelines for the management of acute coronary syndromes in patients presenting without persistent ST-segment elevation: The Task Force for the management of acute coronary syndromes (ACS) in patients presenting without persistent ST-segment elevation of the European Society of Cardiology (ESC). Eur Heart J. 2011;32:2999-3054.

12. Civeira E, del Nogal F, Álvarez AP, Ferrero J, Alcantara AG, Aguado GH, et al. Recomendaciones para el diagnóstico y tratamiento del síndrome coronario agudo sin elevación del segmento ST. Med Intensiva. 2010;34:22-45.

13. Norhammar A, Malmberg K, Rydén L, Tornvall P, Stenestrand U, Wallentin L, Register of Information and Knowledge about Swedish Heart Intensive Care Admission (RIKS-HIA). Under utilisation of evidence-based treatment partially explains for the unfavourable prognosis in diabetic patients with acute myocardial infarction. Eur Heart J. 2003;24:838-44.

14. Norhammar A, Malmberg K, Diderholm E, Lagerqvist B, Lindahl B, Rydén L, et al. Diabetes mellitus: The major risk factor in unstable coronary artery disease even after consideration of the extent of coronary artery disease and benefits of revascularization. J Am Coll Cardiol. 2004;43:585-91.

15. Roa L, Monreal M, Carmona JA, Aguilar E, Coll R, Suárez C. Inercia terapéutica en prevención secundaria de enfermedad cardiovascular. Registro FRENA. Med Clin (Barc). 2010;134:57-63.

16. Anselmino M, Bartnik M, Malmberg K, Rydén L, Euro Heart Survey Investigators. Management of coronary artery disease in patients with and without diabetes mellitus. Acute management reasonable but secondary prevention unacceptably poor: A report from the Euro Heart Survey on Diabetes and the Heart. Eur J Cardiovasc Prev Rehabil. 2007;14:28-36.

17. Dotevall A, Hasdai D, Wallentin L, Battler A, Rosengren A. Diabetes mellitus: Clinical presentation and outcome in men and women with acute coronary syndromes. Data from the Euro Heart Survey ACS. Diabet Med. 2005;22: $1542-50$.

18. Henriksson M, Epstein DM, Palmer SJ, Sculpher MJ, Clayton TC, Pocock SJ, et al The cost-effectiveness of an early interventional strategy in non-ST-elevation acute coronary syndrome based on the RITA 3 trial. Heart. 2008;94:717-23.

19. Subherwal S, Bach RG, Chen AY, Gage BF, Rao SV, Newby LK, et al. Baseline risk of major bleeding in non-ST-segment-elevation myocardial infarction: The CRUSADE (Can Rapid risk stratification of Unstable angina patients Suppress ADverse outcomes with Early implementation of the ACC/AHA Guidelines) Bleeding Score. Circulation. 2009;119:1873-82.

20. Felices-Abad F, Latour-Pérez J, Fuset-Cabanes MP, Ruano-Marco M, Cuñat-de la Hoz J, del Nogal-Sáez F. Indicadores de calidad en el síndrome coronario agudo para el análisis del proceso asistencial pre e intrahospitalario. Med Intensiva. 2010;34:397-417. 


\title{
Accesibilidad al sistema sanitario de los pacientes diabéticos con síndrome coronario agudo con elevación del segmento ST
}

\author{
A. Baeza-Román ${ }^{\text {a,* }}$, E. de Miguel-Balsa ${ }^{a}$, J. Latour-Péreza, V. Díaz de Antoñana-Saez ${ }^{\mathrm{b}}$, \\ J. Arguedas-Cervera ${ }^{c}$, E. Mira-Sánchez ${ }^{\mathrm{d}}$, C.J. Fernández-González ${ }^{\mathrm{e}}$, \\ M. Rico-Sala ${ }^{f}$, M. Lafuente-Mateo ${ }^{g}$ y Grupo ARIAM-SEMICYUC
}

a Unidad de Cuidados Intensivos, Hospital General Universitario de Elche, Elche, España

b Unidad de Cuidados Intensivos, Complejo Hospital San Pedro. Logroño, España

c Unidad de Cuidados Intensivos, Hospital General de Castelló, Castellón. España

d Unidad de Cuidados Intensivos, Hospital General Universitario Reina Sofía. Murcia, España

e Unidad de Cuidados Intensivos, Complejo hospitalario Arquitecto Marcide-Prof. Novoa Santos, Ferrol, España

f Unidad de Cuidados Intensivos, Hospital Arnau de Vilanova, Valencia, España

s Unidad de Cuidados Intensivos, Hospital Nuestra Señora de Gracia, Zaragoza, España

Recibido el 16 de diciembre de 2014; aceptado el 18 de febrero de 2015

Disponible en Internet el 23 de mayo de 2015

\author{
PALABRAS CLAVE \\ Diabetes mellitus; \\ Síndrome coronario \\ agudo con elevación \\ de ST; \\ Retraso; \\ Accesibilidad; \\ Sistema sanitario
}

\begin{abstract}
Resumen
Objetivos: El objetivo de este estudio es medir la accesibilidad al sistema sanitario de los pacientes diabéticos y analizar si las posibles diferencias en la accesibilidad explican la mayor mortalidad conocida en aquellos.

Métodos: Estudio de cohortes retrospectivo, realizado en pacientes diabéticos con síndrome coronario agudo con elevación del segmento ST incluidos en los años 2010 al 2013 del registro ARIAM-SEMICYUC. Se realiza análisis crudo y ajustado mediante regresión logística no condicional.

Resultados: Se han analizado 4817 pacientes, de los cuales 1070 (22,2\%) son diabéticos. Los pacientes diabéticos contactan con el sistema sanitario de la misma forma que los pacientes no diabéticos aunque con mayor retraso (retraso atribuible al paciente 90 min vs. 75 min con $p=0,004$ y retraso prehospitalario $150 \mathrm{~min}$ vs. $130 \mathrm{~min}$ con $\mathrm{p}=0,002)$. Una vez dentro del sistema sanitario, estos pacientes tienen menor tasa de reperfusión ( 50 vs. $57,7 \% ; p<0,001$ ) pero sin objetivar mayor retraso en el tratamiento. Como ya es conocido, los pacientes diabéticos presentan una mayor mortalidad hospitalaria $(12,5$ vs. $6 \%$; $p<0,001)$; sin embargo, no se identifican como variables predictoras independientes de la mortalidad ni el retraso atribuible al paciente ni el retraso prehospitalario.
\end{abstract}

\footnotetext{
* Autor para correspondencia.

Correo electrónico: anna.baeza@coma.es (A. Baeza-Román).

$\diamond$ Los componentes del Grupo ARIAM-SEMICYUC trabajan en Unidades de Cuidados Intensivos/Coronarias de varios hospitales españoles.
} 
Conclusiones: Los pacientes diabéticos tienen una mayor demora en el acceso al sistema sanitario, sin embargo no hemos podido objetivar que esta demora se relacione de forma independiente con la mayor mortalidad.

(c) 2015 Elsevier España, S.L.U. y SEMICYUC. Todos los derechos reservados.

\section{KEYWORDS}

Diabetes mellitus;

Acute coronary

syndrome with

ST-segment

elevation;

Delay;

Access;

Health care

\section{Accessibility to health care of diabetic patients with acute coronary syndrome ST-segment elevation}

\begin{abstract}
Objectives: To measure accessibility to health care among diabetic patients and analyze whether differences in delay explain differences in hospital mortality.

Methods: A retrospective cohort study was conducted in diabetic patients with acute coronary syndrome with ST-segment elevation included in the ARIAM-SEMICYUC registry (2010-2013). Crude and adjusted analyses were performed using unconditional logistic regression.

Results: A total of 4817 patients were analyzed, of whom 1070 (22.2\%) were diabetics.

No differences were found in access to health care between diabetic and non-diabetic patients. Diabetic patients presented with longer patient delay $(90 \mathrm{~min}$ vs. $75 \mathrm{~min} ; \mathrm{p}=.004)$ and prehospital delay $(150 \mathrm{~min}$ vs. $130 \mathrm{~min} ; \mathrm{p}=.002)$. Once the health system was contacted, diabetic patients had a lower reperfusion rate $(50 \%$ vs. $57.7 \% ; p<.001)$, but no longer delay in treatment was observed compared with the non-diabetic individuals. Diabetic patients have greater in-hospital mortality $(12.5$ vs. $6 \%$; $<$ <.001), though neither patient delay nor prehospital delay were identified as independent predictors of in-hospital mortality.

Conclusions: Diabetic patients had a longer delay in access to health care, though such delay was not independently related to increased mortality.

(C) 2015 Elsevier España, S.L.U. and SEMICYUC. All rights reserved.
\end{abstract}

\section{Introducción}

En los pacientes con síndrome coronario agudo con elevación del ST (SCACEST) el tratamiento de reperfusión debe administrarse lo antes posible, y en cualquier caso en las primeras 12 horas tras el inicio de los síntomas ${ }^{1,2}$. En el tiempo total de reperfusión existen básicamente 2 componentes. El tiempo que tarda el paciente en contactar con el sistema sanitario tras el inicio de los síntomas (retraso atribuible al paciente), y el tiempo desde que el paciente contacta y se administra el tratamiento de reperfusión (retraso atribuible al sistema sanitario).

Varios estudios han objetivado que los pacientes diabéticos con SCACEST tardan más en contactar con el sistema sanitario tras el inicio de los síntomas ${ }^{3-7}$, lo cual tiene varias consecuencias. Por un lado, influye directamente en la decisión de realizar reperfusión y en la elección del tratamiento (fibrinólisis vs. intervencionismo coronario percutáneo [ICP]). Por otro lado, el mayor retraso inicial contribuye a un mayor tiempo total de reperfusión y por lo tanto a un mayor tiempo de isquemia miocárdica. Son varios los factores que pueden explicar este mayor retraso de los pacientes diabéticos, sobre todo la sintomatología atípica que presentan ${ }^{3,5,8}$.

El objetivo de este estudio es conocer si existen diferencias en la accesibilidad al sistema sanitario (tiempos de acceso y de reperfusión) de los pacientes diabéticos respecto a los no diabéticos, analizando los diferentes factores que pueden influir en estas diferencias, y por último analizar si explican la mayor mortalidad de estos.

\section{Métodos}

Se trata de un estudio de cohortes retrospectivo basado en los datos de Registro ARIAM-SEMICYUC en la que participan diferentes hospitales españoles ${ }^{9}$. Este registro cumple con la legislación española sobre estudios postautorización de tipo observacional para medicamentos de uso humano (Orden SAS/3470/2009, de 16 de diciembre). Además en mayo de 2012 obtuvo el reconocimiento como Registro de interés para el sistema nacional de salud.

Se han recogido los datos de aquellos pacientes consecutivos que ingresan en la unidad de cuidados intensivos/coronarias con diagnóstico al ingreso de SCACEST, entre los años 2010 y 2013.

Las variables analizadas incluyen datos sociodemográficos (sexo, edad), acceso al sistema sanitario (lugar del primer contacto, forma de traslado al hospital), forma de presentación clínica del SCA (electrocardiograma, síntoma inicial), características clínicas al ingreso (clasificación Killip-Kimball, TIMI, GRACE, CRUSADE) ${ }^{10-12}$ y método de reperfusión.

Respecto a los retrasos al tratamiento se analizan los tempos atribuibles al paciente y al sistema sanitario: a) tiempo atribuible al paciente (desde el inicio de los síntomas hasta el primer contacto médico [PCM]); b) el tiempo prehospitalario (desde el inicio de los síntomas hasta la llegada al hospital, coincidirá con el tiempo atribuible al paciente en el caso de que el PCM se realice en el hospital); c) tiempo de traslado (en aquellos pacientes en los que el PCM no se realiza en el hospital, es el tiempo desde el PCM hasta la 
llegada al hospital); d) tiempo desde la llegada al hospital hasta la administración de fibrinólisis; e) tiempo desde la llegada al hospital hasta la realización de ICP; $f$ ) tiempo total de reperfusión censurado en $12 \mathrm{~h}$ (desde inicio de los síntomas hasta reperfusión). Se explora además mortalidad en unidad de cuidados intensivos, hospitalaria y a los 30 días.

Los datos han sido analizados con SPSS Statistics para Mac, versión 22. En la estadística descriptiva las variables cuantitativas continuas se expresan como la mediana y percentiles 25 y 75, y las variables categóricas como proporciones. La comparación de variables categóricas se realiza con el test ji al cuadrado y la comparación de las variables cuantitativas mediante el test de Mann-Whitney tras objetivar que dichas variables no siguen una distribución normal mediante la prueba de Shapiro-Wilks. Las variables de tiempo se analizan mediante análisis de supervivencia Kaplan Meier: prueba de Breslow. El contraste de hipótesis se realiza de forma bilateral con un nivel de significación alfa del $5 \%$.

Para los análisis de mortalidad se ha utilizado la variable mortalidad hospitalaria, ya que la mortalidad a los 30 días era una variable no obligatoria y presenta muchas pérdidas en la recogida de datos.

Finalmente se realiza una regresión logística binaria para determinar las variables predictoras independientes asociadas a mortalidad hospitalaria. Se incluyen en el modelo de regresión diferentes variables con un nivel de significación menor de 0,1 en el análisis univariante. La asociación entre las variables predictoras y la mortalidad se estima mediante el cálculo de las odds ratio ajustadas y de sus intervalos de confianza de $95 \%$.

\section{Resultados}

En el análisis se incluyen 4685 pacientes con SCACEST, de los cuales el 22,85\% (1070 pacientes) tienen diabetes mellitus.

La tabla 1 recoge el análisis univariante de las principales variables. Los pacientes diabéticos tienen mayor edad que los no diabéticos, y hay mayor proporción de mujeres. El SCA se presenta de forma diferente en los pacientes diabéticos, ya que con más frecuencia se presenta con dolor atípico o indoloro. Sin embargo, la forma de acceso al sistema sanitario es similar en ambos grupos de pacientes siendo el lugar del primer contacto médico más frecuente las urgencias hospitalarias. Tampoco se objetivan diferencias estadísticamente significativas en la forma de traslado al hospital, aunque parece que los pacientes diabéticos tienden a ser trasladados con más frecuencia en transporte sanitario no medicalizado, y menos por ellos mismos o con familiar.

A su llegada a urgencias, los pacientes diabéticos presentan con más frecuencia un electrocardiograma con bloqueo completo de rama izquierda, mayor grado de insuficiencia cardiaca medido por Killip-Kimball, y mayor gravedad medida por TIMI, GRACE y CRUSADE. Tienen además una menor tasa de reperfusión con menor tasa de trombólisis e ICP primaria y una mayor tasa de ICP tardía. También se objetiva una menor tasa de reperfusión en los pacientes diabéticos. La mortalidad en unidad de cuidados intensivos, hospitalaria y a los 30 días también es superior en estos pacientes.

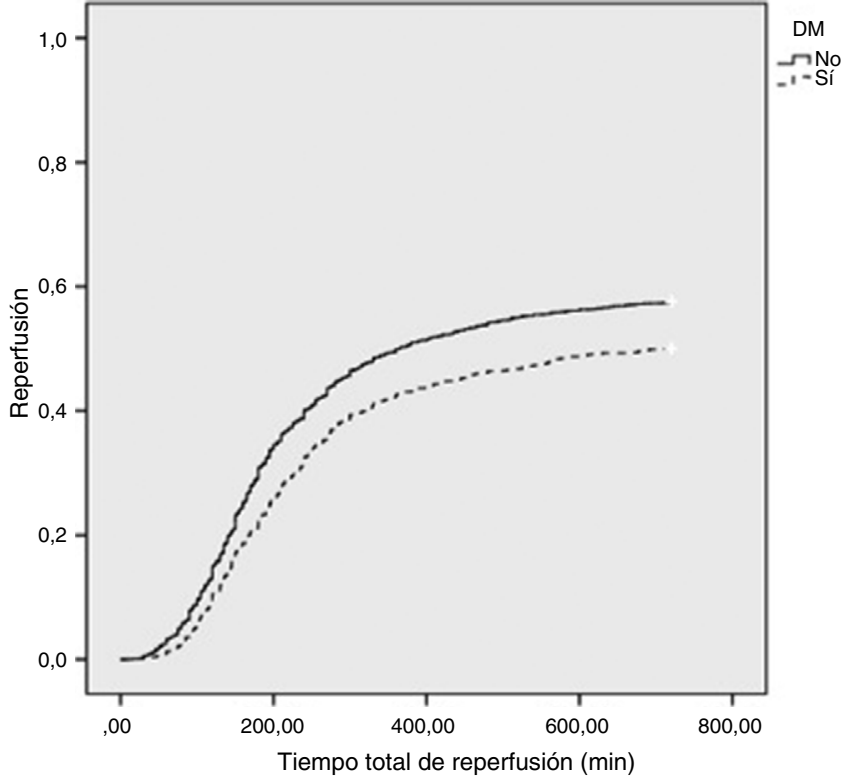

Figura 1 Relación entre el tiempo (min) y reperfusión en pacientes diabéticos y no diabéticos.

La tabla 2 refleja el análisis de los tiempos atribuibles al paciente y atribuibles al sistema sanitario. Los pacientes diabéticos tardan más en realizar el primer contacto médico, lo cual contribuye a un mayor retraso prehospitalario, siendo el tiempo de traslado al hospital similar en ambos grupos. Una vez el paciente ha contactado con el sistema sanitario, no se objetivan diferencias en los tiempos de tratamiento (tiempo urgencias-aguja; tiempo urgencias-balón; tiempo PCM-aguja; tiempo PCM-balón). Todos aquellos tiempos calculados a partir del inicio de los síntomas son mayores en los diabéticos ya que están influenciados por el tiempo atribuible al paciente (tiempo síntomas-aguja; tiempo síntomas-balón; tiempo total de reperfusión).

Se analiza la relación entre el tiempo total de reperfusión y el evento reperfusión, y se objetiva que en los pacientes diabéticos existe una menor probabilidad de ser reperfundido respecto a los no diabéticos (fig. 1).

En el modelo de regresión se introducen las siguientes variables: diabetes mellitus, edad, retraso atribuible al paciente, evento reperfusión, Killip Kimball, TIMI, GRACE, CRUSADE y tiempo total de reperfusión. Se identifican como variables predictoras independientes de mortalidad la diabetes y la gravedad del paciente (TIMI, GRACE y CRUSADE), pero no el retraso atribuible al paciente (tabla 3). Además se aplica la ecuación obtenida con el modelo y se calcula la curva ROC, con un área bajo la curva que es de 0,9 lo cual demuestra muy buen valor predictivo del modelo de regresión.

\section{Discusión}

En nuestro estudio los pacientes diabéticos tienen un mayor retraso atribuible al paciente, y por lo tanto, todos los tiempos que incluyan este intervalo se encuentran aumentados. Diferentes publicaciones ya han mostrado resultados similares. Los estudios de Hasin et al., Woodfield et al., y Goldberg et al., objetivan que en los pacientes diabéticos con SCA 
Tabla 1 Características basales y evolución. Pacientes con SCACEST $(n=4817)$

\begin{tabular}{|c|c|c|c|}
\hline \multirow[t]{2}{*}{ Características basales } & \multicolumn{2}{|c|}{ Pacientes con SCACEST $(n=4817)$} & \multirow[t]{2}{*}{$P$} \\
\hline & DM $22,8 \%(n=1070 / 4685)$ & No DM 77,2\% $(n=3615 / 4685)$ & \\
\hline Edad: & $68,5(60-77)$ & $62(52-74)$ & $<0,001$ \\
\hline Sexo (hombres) $(n=4684)$ & $795(74,3 \%)$ & $2861(79,2 \%)$ & 0,001 \\
\hline \multicolumn{4}{|l|}{ Presentación clínica: } \\
\hline Síntoma inicial $(n=3575)$ & & & 0,003 \\
\hline Indoloro & $42(5,3 \%)$ & $90(3,2 \%)$ & \\
\hline Dolor típico & $674(84,6 \%)$ & $2464(88,7 \%)$ & \\
\hline Dolor atípico & $81(10,2 \%)$ & $224(8,1 \%)$ & \\
\hline Parada cardiorrespiratoria $(n=3640)$ & $37(4,6 \%)$ & $143(5 \%)$ & 0,624 \\
\hline Forma del primer contacto médico $(n=4659)$ & & & 0,237 \\
\hline $061-112$ & $208(19 \%)$ & $693(19,3 \%)$ & \\
\hline Médico & $44(4,1 \%)$ & $162(4,5 \%)$ & \\
\hline Centro salud & $317(29,9 \%)$ & $1105(30,7 \%)$ & \\
\hline Urgencias hospitalarias & $396(37,3 \%)$ & $1359(37,8 \%)$ & \\
\hline Pacientes hospitalizados & $33(3,1 \%)$ & $66(1,8 \%)$ & \\
\hline Otros & $63(5,9 \%)$ & $213(5,9 \%)$ & \\
\hline Transporte $(n=4560)$ & & & 0,055 \\
\hline Paciente-familiar & $469(45,6 \%)$ & $1693(47,9 \%)$ & \\
\hline Transporte sanitario no medicalizado & $80(7,8 \%)$ & $196(5,5)$ & \\
\hline $061-112$ & $394(38,3 \%)$ & $1363(38,6 \%)$ & \\
\hline Otros & $85(8,3 \%)$ & $280(7,9 \%)$ & \\
\hline ECG al ingreso $(n=4589)$ & & & $<0,001$ \\
\hline Elevación $S T>2 \mathrm{~mm}$ en $>2$ derivaciones & 787 (75\%) & $2787(78,7 \%)$ & \\
\hline Elevación $\mathrm{ST}<2 \mathrm{~mm} 0<2$ derivaciones & $230(21,9 \%)$ & $709(20 \%)$ & \\
\hline Nuevo BCRIHH & $32(3,1 \%)$ & $44(1,2 \%)$ & \\
\hline \multicolumn{4}{|l|}{ Gravedad al ingreso: } \\
\hline KK inicial $(n=4634)$ & & & $<0,001$ \\
\hline $1-2$ & $923(87,1 \%)$ & $3333(93,3 \%)$ & \\
\hline $3-4$ & $137(12,9 \%)$ & $241(6,7 \%)$ & \\
\hline $\operatorname{TIMI}(n=3466)$ & & & $<0,001$ \\
\hline$<4$ & $266(34,5 \%)$ & $1292(48 \%)$ & \\
\hline$\geq 4$ & $506(65 \%)$ & $1402(52 \%)$ & \\
\hline GRACE $(n=4635)$ & $160(139-185)$ & $146(125-172)$ & $<0,001$ \\
\hline CRUSADE $(n=4645)$ & $47(29-57)$ & $37(20-49)$ & $<0,001$ \\
\hline Tasa de reperfusión $(n=4666)$ & $534(50 \%)$ & $3599(57,7 \%)$ & $<0,001$ \\
\hline Método reperfusión $(n=4666)$ & & & $<0,001$ \\
\hline No realizada & $194(18,2 \%)$ & $525(14,6 \%)$ & \\
\hline Trombólisis & $271(25,4 \%)$ & $1067(29,6 \%)$ & \\
\hline ICP primaria & $463(43,4 \%)$ & $1635(45,4 \%)$ & \\
\hline ICP tardía & $139(13 \%)$ & $372(10,3 \%)$ & \\
\hline \multicolumn{4}{|l|}{ Mortalidad: } \\
\hline En UCI $(n=4515)$ & $82(8 \%)$ & $121(3,5 \%)$ & $<0,001$ \\
\hline Hospitalaria $(n=3746)$ & $109(12,5 \%)$ & $175(6 \%)$ & $<0,001$ \\
\hline A los 30 días $(n=2634)$ & $116(19,2 \%)$ & $201(9,9 \%)$ & $<0,001$ \\
\hline
\end{tabular}

BCRIHH: bloque completo rama izquierda; DM: diabetes emllitus; ECG: electrocardiograma; ICP: intervencionismo coronario percutáneo; KK: Killip-kimball; SCACEST: síndrome coronario agudo con elevación del ST; UCI: Unidad de Cuidados Intensivos.

el tiempo entre la aparición de los síntomas y el tratamiento es más elevado que en los pacientes no diabéticos ${ }^{3-5}$. Ribeiro et al., analizan los pacientes con SCACEST en función del retraso prehospitalario, y objetivan que en el grupo de pacientes con mayor retraso existe una mayor proporción de diabéticos, y además, identifican la diabetes como una variable predictora independiente asociada a un retraso prehospitalario mayor a 3 horas $^{6}$. Goolberg et al., en un estudio posterior al que ya se ha mencionado, analizan datos de 14 países entre los cuales está España (Países de Norte y Sudamérica, Europa, Australia y Nueva Zelanda) y objetivan que en todas las áreas analizadas los pacientes diabéticos tienen un mayor retraso prehospitalario ${ }^{7}$. Sin embargo, en ninguna de estas publicaciones se analiza la posible relación entre este mayor retraso prehospitalario de los pacientes diabéticos y su mayor mortalidad. En nuestro estudio, la 
Tabla 2 Tiempos de retraso atribuible al paciente y retraso atribuible al sistema sanitario. Expresados en minutos con medianas y rango intercuartil

\begin{tabular}{|c|c|c|c|}
\hline \multirow[t]{2}{*}{ Tiempos } & \multicolumn{2}{|c|}{ Pacientes con SCACEST $(n=4817)$} & \multirow[t]{2}{*}{$P$} \\
\hline & DM 22,85\% $(n=1070 / 4685)$ & No DM $77,17 \%(n=3615 / 4685)$ & \\
\hline Retraso paciente $(n=3382)$ & $90(40-210)$ & $75(31-183)$ & 0,004 \\
\hline Retraso prehospitalario $(n=3709)$ & $150(82-284)$ & $130(72-267)$ & 0,002 \\
\hline Retraso transporte $(n=1879)$ & $57(30-90)$ & $53(26-86)$ & 0,18 \\
\hline Tiempo síntomas-aguja $(n=1297)$ & $180(120-270)$ & $150(100-250)$ & 0,005 \\
\hline Tiempo PCM-aguja $(\mathrm{n}=934)$ & $80(50-128)$ & $70(40-115)$ & 0,115 \\
\hline Tiempo urgencias-aguja $(n=1003)$ & $49(30-84)$ & $42(28-75)$ & 0,126 \\
\hline Tiempo síntomas-balón $(n=2554)$ & $450(210-1956)$ & $375(190-1560)$ & 0,012 \\
\hline Tiempo PCM-balón $(n=1413)$ & $134(95-225)$ & $125(90-196)$ & 0,079 \\
\hline Tiempo urgencias-balon $(n=1227)$ & $113(70-192)$ & $105(62-169)$ & 0,065 \\
\hline Tiempo total de reperfusión $(n=2608)$ & $195(135-285)$ & $175(120-270)$ & 0,002 \\
\hline
\end{tabular}

DM: diabetes mellitus; PCM: primer contacto médico; SCACEST: síndrome coronario agudo con elevación del segmento ST.

accesibilidad al sistema sanitario es similar en pacientes diabéticos y no diabéticos, y una vez los pacientes han entrado en contacto con el sistema sanitario, los tiempos calculados son similares en ambos grupos. En el año 2002, ColmeneroRuiz et al., analizan las diferencias en el manejo de los pacientes con SCA entre las diferentes comunidades autónomas españolas a partir del registro ARIAM, y objetivan que existen diferencias en la forma de acceso al sistema sanitario entre las diferentes comunidades estudiadas, sin que esto se traduzca en diferencias de mortalidad. Existe también una importante variabilidad en el porcentaje de pacientes diabéticos entre comunidades, sin embargo no se estudia la posible asociación entre la diabetes y el modo de acceso ${ }^{13}$. Otro hallazgo en nuestro estudio es que los pacientes diabéticos presentan más frecuentemente síntomas atípicos o indoloros lo cual puede explicar el mayor retraso en solicitar ayuda, hecho que se ha descrito en estudio previos ${ }^{5}$. Leslie et al. analiza en su publicación las diferentes causas de retraso prehospitalario en pacientes con SCA, siendo la causa más frecuente el mal reconocimiento de los síntomas y no conocer la gravedad del proceso ${ }^{8}$. En nuestro estudio se objetiva además, que los pacientes diabéticos tienen una menor tasa de reperfusión y una mayor mortalidad, lo cual está también descrito previamente en la literatura ${ }^{14}$.

Nuestro estudio no ha podido objetivar una asociación independiente del retraso atribuible al paciente con la mortalidad. Nuestros análisis concluyen que las variables que se asocian de manera independiente con la mortalidad en

Tabla 3 Modelo de regresión logística binaria

\begin{tabular}{llr}
\hline Variables predictoras & OR (IC 95\%) & \multicolumn{1}{c}{$\mathrm{p}$} \\
\hline $\begin{array}{l}\text { Retraso } \\
\quad \text { atribuible al }\end{array}$ & $1,017(0,995-1,041)$ & 0,129 \\
$\quad$ paciente $(\mathrm{en}$ & & \\
$\quad$ horas) & $1,51(1,046-2,181)$ & 0,028 \\
DM & $2,314(1,106-4,841)$ & 0,026 \\
TIMI & $1,031(1,025-1,036)$ & $<0,001$ \\
GRACE & $1,031(1,017-1,045)$ & $<0,001$ \\
CRUSADE &
\end{tabular}

DM: diabetes mellitus; IC: intervalo de confianza; OR: odds ratio. los pacientes con SCACEST son en su mayor gravedad inicial medidas por TIMI, GRACE y CRUSADE, así como el hecho de padecer diabetes mellitus.

La limitación más importante de nuestro estudio es el seguimiento a partir del alta hospitalaria. Para el análisis de mortalidad hemos decidido utilizar la variable de mortalidad hospitalaria debido al elevado número de pérdidas en la variable de mortalidad a los 30 días, aunque consideramos también que dichas pérdidas son aleatorias, por lo que no sesga nuestros resultados.

Por otro lado se trata de un estudio multicéntrico en el que participan centros de toda España con lo que se obtiene una muestra bien representativa de la población española.

\section{Conclusión}

Los pacientes diabéticos tardan más en contactar con el sistema sanitario, sin embargo, lo que parece que determina su mayor mortalidad no es este retraso, si no el hecho de padecer diabetes y la mayor gravedad que presentan al ingreso hospitalario.

\section{Conflicto de intereses}

Los autores declaran no tener ningún conflicto de intereses.

\section{Bibliografía}

1. O'Gara PT, Kushner FG, Ascheim DD, Casey DE Jr, Chung MK, et al., American College of Emergency Physicians, Society for Cardiovascular Angiography and Interventions. 2013 ACCF/AHA guideline for the management of ST-elevation myocardial infarction: a report of the American College of Cardiology Foundation/American Heart Association Task Force on Practice Guidelines. J Am Coll Cardiol. 2013;61:e78-140.

2. Steg PG, James SK, Atar D, Badano LP, Blömstrom-Lundqvist C, Borger MA, et al., Task force on the management of STsegment elevation acute myocardial infarction of the European Society of Cardiology (ESC). ESC Guidelines for the management of acute mmyocardial infarction in patients presenting with ST-segment elevation. JT Eur Heart J. 2012;33:2569-619. 
3. Hasin T, Hochadel M, Gitt AK, Behar S, Bueno H, Hasin Y. Comparison of treatment and outcome of acute coronary syndrome in patients with versus patients without diabetes mellitus. Am J Cardiol. 2009;103:772-8.

4. Woodfield SL, Lundergan CF, Reiner JS, Greenhouse SW, Thompson MA, Rohrbeck SC, et al. Angiographic findings and outcome in diabetic patients treated with thrombolytic therapy for acute mmyocardial infarction: The GUSTO-I Experience. J Am Coll Cardiol. 1996;28:1661-9.

5. Goldberg RJ, Steg PG, Sadiq I, Granger GB, Jackson EA, Budaj A, et al. Extent of, and factors associated with, delay to hospital presentation in patients with acute coronary disease (the GRACE Registry). Am J Cardiol. 2002;89:791-6.

6. Ribeiro S, Gaspar A, Rocha S, Nabais S, Azevedo P, Salgado $A$, et al. Predictors of pre-hospital delay in patients with ST-segment elevation myocardial infarction. Rev Port Cardiol. 2010;29:1521-32.

7. Goldberg RJ, Spencer FA, Fox KA, Brieger D, Steg G, Gurfinkel E, et al. Prehospital delay in patients with acute coronary syndromes (from the Global Registry of Acute Coronary Events [GRACE]). Am J Cardiol. 2009;103:598-603.

8. Leslie WS, Urie A, Hooper J, Morrison CE. Delay in calling for help during myocardial infarction: Reasons for the delay and subsequent pattern of accessing care. Heart. 2000;84: 137-41.

9. Latour-Pérez J, Fuset-Cabanes MP, Ruano-Marco M, del NogalSáez F, Cunat-de la Hoz J, Alcoverro-Pedrola JM, et al. ARIAM
Análisis del corte 2013. SEMICYUC: Sociedad Española de Medicina Intensiva y Unidades Coronarias. 2013.

10. Morrow DA, Antman EM, Charlesworth A, Cairns R, Murphy SA, de Lemos JA, et al. TIMI Risk score for ST-elevation myocardial infarction: a convenient, bedside, clinical score for risk assessment at presentation: an intravenous nPA for treatment of infarcting myocardium early II Trial Substudy. Circulation. 2000;102:2031-7.

11. Fox KAA, Dabbous OH, Goldberg RJ, Pieper KS, Eagle KA, van de Werf $F$, et al. Prediction of risk of death and myocardial infarction in the six months after presentation with acute coronary syndrome: prospective multinational observational study (GRACE). BMJ. 2006;333:1091.

12. Subherwal S, Bach RG, Chen AY, Gage BF, Rao SV, Newby LK, et al. Baseline risk of major bleeding in non-ST-segmentelevation myocardial infarction: the CRUSADE (Can rapid risk stratification of unstable angina patients suppress adverse outcomes with early implementation of the ACC/AHA Guidelines) bleeding score. Circulation. 2009;119:1873-82.

13. Colmenero-Ruiz M, Reina-Toral A, Jimenez-Quintana $M M$, Aguayo-de-Hoyos E, Ruiz-Bailén M, García-Alcántara A, et al. Variabilidad en el manejo del infarto agudo de miocardio en el registro ARIAM en el año 2002. Med Intensiva. 2005;29:420-9.

14. Nauta ST, Deckers JW, Akkerhuis KM, van Domburg RT. Shortlong-term mortality after myocardial infarction in patients with and without diabetes: changes from 1985 to 2008. Diab Care. 2012;35:2043-7. 


\section{CARTA A MEDICINA INTENSIVA Y REPLICA A LOS AUTORES I:}

\section{MEDINTENSIVA-D-16-00087 \\ "COMENTARIOS AL ARTÍCULO: Accesibilidad al sistema sanitario de los pacientes diabéticos con síndrome coronario agudo con elevación del segmento ST”.}

Diego Fernández-Rodríguez, M.D (a); María Facenda-Lorenzo, M.D. (a); Julio Hernández- Afonso, Ph.D (a); Francisco Bosa-Ojeda, Ph.D (b).

a.- Servicio de Cardiología, Hospital Universitario Nuestra Señora de Candelaria (Universidad de La Laguna), Santa Cruz de Tenerife, España.

b.- Servicio de Cardiología, Hospital Universitario de Canarias (Universidad de La Laguna), San Cristóbal de La Laguna, España.

Felicitamos a Baeza-Román et $\mathrm{al}^{(1)}$ por su interesante trabajo sobre la accesibilidad al sistema sanitario de pacientes con diabetes mellitus (DM) y síndrome coronario agudo con elevación del segmento ST (SCACEST). El trabajo se basó en un análisis retrospectivo del Registro ARIAM-SEMICYUC (4.817 pacientes consecutivos admitidos en múltiples instituciones españolas durante el periodo 2010-2013) que fue analizado según la presencia de DM. El análisis multivariante objetivó que la DM era un predictor independiente de mortalidad hospitalaria. Asimismo, se observó que los diabéticos presentaban mayor "retraso atribuible al paciente" lo cual aumentaba el tiempo total hasta la reperfusión. No obstante, el "retraso atribuible al paciente" no se mostró como predictor independiente de mortalidad hospitalaria.

El principal interés del estudiol consiste en mostrar, mediante una población amplia durante lapso temporal extenso, que los diabéticos presentan dificultades para acceder al sistema sanitario. Sin embargo, el análisis merece ciertas consideraciones:

- "Variables confusoras" y Demora: Debido al diseño del Registro ARIAMSEMICYUC

1, no constan relevantes características basales (ej: infarto de miocardio previo...). Asimismo, ambos grupos presentan diferencias significativas en otras características basales como edad, género o presentación clínica. Dado que posibles "variables confusoras" no se recogieron y tampoco se realizó un análisis multivariante para evitar la influencia de las que sí fueron recogidas, no se puede concluir firmemente que las diferencias en el "retraso atribuible al paciente" o el tiempo total hasta la reperfusión estén mediadas únicamente por la DM.

- "Redes asistenciales" y Demora: El beneficio de la reperfusión depende del tiempo. La implementación de redes de asistencia al SCACEST permitió reducir los retrasos en la atención médica, atribuibles tanto al sistema como a los pacientes ${ }^{(2)}$. El "retraso atribuible al sistema" fue reducido mediante el establecimiento de un sistema de ambulancias con personal capacitado para proporcionar atención médica y realizar electrocardiogramas domiciliarios, activar a distancia a los equipos de hemodinámica y trasladar directamente a los pacientes al laboratorio de hemodinámica. En cuanto al "retraso atribuible al paciente", se disminuyó mediante la implantación de números telefónicos específicos para recibir alertas extrahospitalarias de particulares y centros coordinadores que remitían ambulancias con personal entrenado en caso de sospechar un SCACEST (2),(3). Asimismo, se ha sugerido que las campañas de concienciación pública podrían, además de aumentar el número de pacientes que contactan con la red, reducir la demora en acceder al sistema ${ }^{(4)}$.

- "Redes asistenciales" y Reperfusión: Las redes asistenciales, que se empezaban a instaurar en España durante el periodo del estudio ${ }^{(5)}$, permitieron minimizar el porcentaje de pacientes que no recibían terapia de reperfusión en el SCACEST, lo que 
unido a la reducción de los tiempos en la asistencia mejoró el pronóstico de los pacientes $^{(2)}$. Sin embargo, en este trabajo destaca un porcentaje muy elevado de pacientes sin reperfusión, especialmente entre los diabéticos.

En conclusión, consideramos que realizar un análisis multivariante específico de los intervalos temporales y ampliar la información, si estuviera disponible, sobre las redes asistenciales al SCACEST podría mejorar la comprensión del impacto de la diabetes sobre la accesibilidad al sistema sanitario, la reperfusión y la mortalidad.

\section{BIBLIOGRAFÍA.}

1.- Baeza-Román A, de Miguel-Balsa E, Latour-Pérez J, Díaz de Antoñana-Saez V, Arguedas-Cervera J, Mira-Sánchez E, et al. Accessibility to health care of diabetic patients with acute coronary syndrome ST-segment elevation. Med Intensiva. 2016;40:90-95.

2.- Steg PG, James SK, Atar D, Badano LP, Blömstrom-Lundqvist C, Borger MA, et al. ESC Guidelines for the management of acute myocardial infarction in patients resenting with ST-segment elevation. Eur Heart J. 2012;33:2569-619.

3.- Goldberg RJ, Steg PG, Sadiq I, Granger CB, Jackson EA, Budaj A, Brieger D, Avezum A, Goodman S. Extent of, and factors associated with, delay to hospital presentation in patients with acute coronary disease (the GRACE registry). Am J Cardiol. 2002;89:791-6.

4.- Regueiro A, Rosas A, Kaifoszova Z, Faixedas MT, Curos A, Tresserras R, et al. Impact of the "ACT NOW. SAVE A LIFE" public awareness campaign on the performance of a European STEMI network. Int J Cardiol. 2015;197:110-2.

5.- Regueiro A, Tresserras R, Goicolea J, Fernández-Ortiz A, Macaya C, Sabaté M. Primary percutaneous coronary intervention: models of intervention in Spain. EuroIntervention. 2012;8 Suppl P:P90-3. 


\section{Respuesta de los autores: Accesibilidad al sistema sanitario de los pacientes diabéticos con síndrome coronario agudo con elevación del segmento ST}

\section{Author's reply: Accessibility to healthcare of diabetic patients with acute coronary syndrome with ST-segment elevation}

\section{Sr. Director:}

Agradecemos a Fernández-Rodríguez et al. ${ }^{1}$, el interés mostrado por nuestro artículo, y las consideraciones realizadas, así como la oportunidad de clarificar algunos puntos que pueden haber sido mal interpretados.

Fernández-Rodríguez et al. ${ }^{1}$ sugieren la existencia de un posible sesgo de confusión debido a un insuficiente control de las variables. En este punto hay que decir que el registro ARIAM-SEMICYUC consta de múltiples variables, entre las cuales se encuentran incluidas todas las que se han mencionado en su carta. Sin embargo, tal como se indica en el artículo, el objetivo de nuestro estudio no era estudiar las causas de los retrasos existentes en la atención al paciente con SCACEST, sino cuantificarlos y estudiar su posible impacto sobre la mortalidad. En consecuencia, y siguiendo una estrategia parsimoniosa, nuestro modelo multivariante evitó incluir las variables superfluas para estos objetivos $^{2}$

Estamos de acuerdo en que la implantación de las redes asistenciales ha permitido mejorar los tiempos en la atención médica al SCA. Sin embargo, no se ha demostrado que estas redes hayan conseguido reducir de forma efectiva el retraso atribuible al paciente, tal y como se objetiva en el trabajo citado de Regueiro et al., en el que, tras una intervención comunitaria, en la que se incluían campañas de concienciación comunitaria, no se consiguió una reducción significativa de dicho retraso ${ }^{3}$. Goldberg et al., en el trabajo citado por ustedes, igualmente encontraba una mayor tasa de pacientes diabéticos en los grupos con mayor retraso prehospitalario $^{4}$.

Efectivamente nuestro trabajo objetiva una baja tasa de reperfusión entre los pacientes diabéticos. Las principales guías del manejo del SCA consideran al paciente diabético de alto riesgo, y recomiendan un manejo agresivo farmacológico e intervencionista, similar a los pacientes de alto riesgo no diabéticos. Sin embargo, diferentes registros han mostrado una infrautilización del tratamiento basado en la evidencia en los pacientes diabéticos. En un trabajo previo, pudimos objetivar esta infrautilización del tratamiento de revascularización entre los diabéticos con SCASEST en España, y sugeríamos que detrás de las causas que motivaban el manejo conservador en estos pacientes, estaba el temor a las posibles complicaciones hemorrágicas, y a la presencia de lesiones coronarias avanzadas ${ }^{5}$.

Como conclusión, podemos afirmar que los pacientes diabéticos continúan teniendo un mayor retraso atribuible al paciente, a pesar de las redes asistenciales implementadas en España. Las causas de este mayor retraso, así como las causas de la baja tasa de reperfusión en los pacientes diabéticos con SCACEST, no son bien conocidas, por lo que debe ser pregunta de estudio en futuros trabajos.

\section{Bibliografía}

1. Fernández-Rodríguez D, Facenda-Lorenzo M, Hernández-Afonso J, Bosa-Ojeda FPD. Comentarios al artículo: Accesibilidad al sistema sanitario de los pacientes diabéticos con síndrome coronario agudo con elevación del segmento ST. Med Intensiva. 2016;40:522.

2. Núñez E, Steyerberg EW, Núñez J. Regression modeling strategies [Article in Spanish]. Rev Esp Cardiol. 2011;64:501-7.

3. Regueiro A, Rosas A, Kaifoszova Z, Faixedas MT, Curos A, Tresserras R, et al. Impact of the "ACT NOW. SAVE A LIFE" public awareness campaign on the performance of a European STEMI network. Int J Cardiol. 2015;197:110-2.

4. Goldberg RJ, Steg PG, Sadiq I, Granger CB, Jackson EA, Budaj A, et al. Extent of, and factors associated with, delay to hospital presentation in patients with acute coronary disease (the GRACE registry). Am J Cardiol. 2002;89:791-6.

5. Baeza Román A, Latour Pérez J, de Miguel Balsa E, Pino Izquierdo K, Coves Orts FJ, García Ochando L, et al. Early invasive strategy in diabetic patients with non-ST-segment elevation acute coronary syndromes [Article in Spanish]. Med Clin (Barc). 2014;142:427-31.

A. Baeza-Román*, J. Latour-Pérez y E. de Miguel-Balsa

Unidad de Cuidados Intensivos, Hospital General

Universitario de Elche, Elche, Alicante, España

* Autor para correspondencia.

Correo electrónico: anna.baeza@coma.es

(A. Baeza-Román).

http://dx.doi.org/10.1016/j.medin.2016.05.010 


\section{CARTA A MEDICINA INTENSIVA Y REPLICA A LOS AUTORES II:}

MEDINTENSIVA-D-16-00091

"COMENTARIOS AL ARTÍCULO: Accesibilidad al sistema sanitario de los pacientes diabéticos con síndrome coronario agudo con elevación del segmento $S T$ : Educación y concienciación en el paciente diabético con síndrome coronario agudo con elevación del ST".

Llopis-García G, Rodríguez-Adrada E, González-Del Castillo J,Martín-Sánchez FJ.

\section{Estimado Sr Director,}

Hemos leído con interés el artículo de A. Baeza Román et al donde se afirma que los pacientes diabéticos con síndrome coronario agudo con elevación del segmento ST (SCACEST), en comparación con los no diabéticos, tiene una mayor demora en el acceso al sistema sanitario aunque no se relaciona con una mayor mortalidad intrahospitalaria $^{(1)}$. En este sentido y de cara a la interpretación de los resultados nos gustaría realizar algunos comentarios.

En primer lugar, los autores dicen que el retraso del paciente podría justificarse con el antecedente de diabetes mellitus, hecho descrito ampliamente en la literatura. Sin embargo, hubiera sido de interés conocer el efecto de la mayor edad y frecuencia de sexo femenino en este grupo de población, ya que son también características que pueden influir en este hecho ${ }^{(2)}$. Además, el retraso puede condicionar la toma de decisión sobre la realización o no de la reperfusión y en la selección de la mejor técnica (angioplastia primaria frente a fibrinolisis) ${ }^{(3)}$.

En segundo lugar, el trabajo muestra que el antecedente de diabetes se asocia de forma estadísticamente significativa con una evaluación más compleja, al tener más frecuencia de bloqueo de rama izquierda en el electrocardiograma, mayor gravedad según las escalas Killip, Grace y TIMI, riesgo de sangrado según la escala CRUSADE y menor frecuencia de recibir tratamiento de reperfusión. Además, los autores no aportan datos sobre el tratamiento farmacológico recibido en la primera atención. En este sentido, numerosos estudios han demostrado la mayor eficacia de la doble antiagregración con los nuevos antiagregantes, como el ticagrelor y el prasugrel, en dicho grupo de pacientes en comparación con el clopidogrel ${ }^{(2)}$.

En tercer lugar, los autores aportan exclusivamente datos de mortalidad intrahospitalaria pero no de mortalidad previa a la llegada al hospital ni tras el alta a corto, medio o largo plazo, con lo cual la influencia de estos factores no puede ser analizada.

Teniendo en cuenta lo anteriormente escrito, y considerando las posibles limitaciones del presente trabajo, creemos que debemos continuar mejorando aspectos de la educación del paciente diabético a la hora de reconocer posibles síntomas y signos relacionados con el infarto agudo de miocardio, de cara a minimizar los tiempos de aviso o consulta a los servicios de urgencias ${ }^{(4)}$. Así mismo, insistir en la concienciación del médico responsable de la primera atención ante la presencia de diabetes mellitus ${ }^{(5)}$, ya que podría tener un efecto no cuantificado en el tratamiento inicial del SCACEST y en los resultados previos o al alta de la hospitalización. 


\section{BIBLIOGRAFÍA}

1.-Baeza-Román A, de Miguel-Balsa E, Latour-Pérez J, Díaz de Antoñana-Saez V, Arguedas- Cervera J, Mira-Sánchez E, et al. Accesibilidad al sistema sanitario de los pacientes diabéticos con síndrome coronario agudo con elevación del segmento ST. Med Intensiva. 2016;40:90-5.

2.-Steg G, James S, Atar D, Badano L, Blömstrom-Lundqvist C, Borger M, et al. ESC Guidelines for the management of acute myocardial infarction in patients presenting with ST-segment elevation. Eur Heart J. 2012;33:2569-617.

3.-Reina Toral A, Colmenero Ruiz M, García Pérez C, Expósito Ruiz M, De Antonio Martín E, Bermúdez Tamayo C, et al. Diferencias en los resultados de la atención a los pacientes con síndrome coronario agudo con elevación del segmento ST (SCACEST) en función del acceso inicial a hospitales con o sin sala de hemodinámica en Andalucía. Emergencias. 2014;26:101-8.

4.-Peters A, Laffel L; American Diabetes Association Transitions Working Group. Diabetes care for emerging adults: recommendations for transition from pediatric to adult diabetes care systems: a position statement of the American Diabetes Association, with representation by the American College of Osteopathic Family Physicians, the American Academy of Pediatrics, the American Association of Clinical Endocrinologists, the American Osteopathic Association, the Centers for Disease Control and Prevention, Children with Diabetes, The Endocrine Society, the International Society for Pediatric and Adolescent Diabetes, Juvenile Diabetes Research Foundation International, the National Diabetes Education Program, and the Pediatric Endocrine Society (formerly Lawson Wilkins Pediatric Endocrine Society). Diabetes Care. 2011;34:2477-85.

5.-Armstrong PW, Gershlick AH, Goldstein P, Wilcox R, Danays T, Lambert Y, et al. Fibrinolysis or primary PCI in ST-segment elevation myocardial infarction. N Engl J Med. 2013;368:1379-87 
Education Program, and the Pediatric Endocrine Society (formerly Lawson Wilkins Pediatric Endocrine Society). Diabetes Care. 2011;34:2477-85.

5. Armstrong PW, Gershlick AH, Goldstein P, Wilcox R, Danays T, Lambert $\mathrm{Y}$, et al. Fibrinolysis or primary $\mathrm{PCl}$ in $\mathrm{ST}$-segment elevation myocardial infarction. N Engl J Med. 2013;368: 1379-87.

G. Llopis-García ${ }^{a, *}$, E. Rodríguez-Adrada ${ }^{a}$,

J. González-del Castillo ${ }^{a, b}$ y F.J. Martín-Sánchez ${ }^{a, b}$ a Servicio de Urgencias, Hospital Clínico San Carlos, Instituto de Investigación Sanitaria del Hospital Clínico San Carlos (IdISSC), Madrid, España

${ }^{\mathrm{b}}$ Facultad de Medicina, Universidad Complutense de Madrid, Madrid, España

* Autor para correspondencia.

Correo electrónico: fjjms@hotmail.com (G. Llopis-García).

http://dx.doi.org/10.1016/j.medin.2016.04.009

\section{Respuesta de los autores: Educación y concienciación en el paciente diabético con síndrome coronario agudo con elevación del ST}

\section{Author's reply: Education and awareness in the diabetic patient with ST-segment elevation acute coronary syndrome}

\section{Sr. Director:}

Agradecer en primer lugar a Llopis-García et al. ${ }^{1}$. el interés mostrado por nuestro artículo ${ }^{2}$, sobre todo con relación a las causas del retraso de los pacientes diabéticos con síndrome coronario agudo, un tema sobre el que existe una amplia bibliografía. Sin embargo, queremos insistir en el hecho de que nuestro estudio no trata de las causas del retraso. El objetivo principal era cuantificar su magnitud y las posibles consecuencias de ese retraso sobre la mortalidad hospitalaria. En consecuencia, los comentarios vertidos por Llopis-García et al. guardan poca relación con nuestro trabajo. A pesar ello nos gustaría realizar algunas aclaraciones.

Respecto a la primera consideración, teniendo en cuenta el objetivo del estudio, no hemos considerado necesario realizar una comparación de las características basales de los pacientes diabéticos frente a los no diabéticos que, además, como bien han comentado en su carta, las múltiples diferencias existentes están bien descritas en la literatura $^{3}$. Por el mismo motivo, y respecto a la segunda consideración, tampoco hemos aportado información sobre el tratamiento administrado en la primera atención médica, a pesar de que sí que se encuentra disponible en el registro ARIAM-SEMICYUC. Sí que se aporta información respecto a cuál era la forma de acceso al sistema sanitario (Urgencias, llamada a 112...), sin que hayamos encontrado diferencias estadísticamente significativas entre los paciente con y sin diabetes.

Respecto a la última consideración, una de las limitaciones más importantes de nuestro estudio es el seguimiento a partir del alta hospitalaria. En el primer análisis univariante, se objetiva una mayor tasa de mortalidad en los pacientes diabéticos tanto en la $\mathrm{UCl}$, como al alta hospitalaria, como al seguimiento posterior a los 30 días. Sin embargo, para realizar el modelo de regresión logístico y para identificar las variables independientemente asociadas a la mortalidad, se decidió utilizar la mortalidad hospitalaria, debido a que la mortalidad a los 30 días es una variable no obligatoria en el registro ARIAM-SEMICYUC y presenta muchas pérdidas en la recogida de datos, aunque consideramos también que dichas pérdidas son aleatorias, por lo que no sesga nuestros resultados.

Dicho esto, nuestro estudio no ha podido objetivar una asociación independiente con la mortalidad, ni del retraso atribuible al paciente, ni de la menor tasa de reperfusión encontrada, pero sí, con la mayor gravedad objetivada de los diabéticos, medida por TIMI, GRACE y CRUSADE, así como el propio hecho de padecer diabetes mellitus.

Para concluir, dado que no se encontraron diferencias respecto a los tiempos de atención médica, una vez los pacientes habían contactado con el sistema sanitario, estamos de acuerdo en que para mejorar los resultados de los pacientes diabéticos con síndrome coronario agudo (SCA), gran parte de los esfuerzos deben centrarse en la educación del propio paciente, así como en seguir investigando respecto a las causas que motivan el mayor retraso atribuible al mismo y sobre las causas de su peor evolución, puesto que, a pesar de los avances en el manejo del SCA, no se ha encontrado una reducción significativa de la mortalidad respecto a los pacientes no diabéticos ${ }^{4}$.

\section{Bibliografía}

1. Llopis-García G, Rodríguez-Adrada E, González-Del Castillo J, Martín-Sánchez FJ. Education and awareness in the diabetic patient with ST-segment elevation acute coronary syndrome [Article in English, Spanish]. Med Intensiva. 2016. pii: S0210-5691(16)30065-1. doi: 10.1016/j.medin.2016.04.009. [Epub ahead of print].

2. Baeza-Román A, de Miguel-Balsa E, Latour-Pérez J, Díaz de Antoñana-Saez V, Arguedas-Cervera J, Mira-Sánchez E, et al. Accessibility to health care of diabetic patients with acute coronary syndrome ST-segment elevation. Med Intensiva. 2016;40:90-5.

3. Donahoe SM, Stewart GC, McCabe CH, Mohanavelu S, Murphy SA, Cannon CP, et al. Diabetes and mortality following acute coronary syndromes. JAMA. 2007;298:765-75.

4. Fox CS, Coady S, Sorlie PD, Levy D, Meigs JB, D'Agostino RB, et al. Trends in cardiovascular complications of diabetes. JAMA. 2004;292:2495-9. 
A. Baeza-Román*, E. de Miguel-Balsa y J. Latour-Pérez

Unidad de Cuidados Intensivos, Hospital General Universitario de Elche, Elche, Alicante, España
* Autor para correspondencia.

Correo electrónico: anna.baeza@coma.es (A. Baeza-Román).

http://dx.doi.org/10.1016/j.medin.2016.06.003 


\title{
PODER PREDICTIVO DEL SCORE GRACE EN LA POBLACIÓN DIABÉTICA ESPAÑOLA.
}

\begin{abstract}
:
Introducción: La estratificación del riesgo al ingreso de los pacientes con síndrome coronario agudo (SCA) se recomienda en el manejo inicial. La diabetes mellitus (DM) se ha identificado como predictor independiente de mortalidad en pacientes con SCA, sin embargo no está incluida en la principal escala de riesgo isquémico, el score GRACE.

Objetivos: El objetivo de este estudio es comprobar la validez de la escala de riesgo GRACE en la población actual, y concretamente en el subgrupo de pacientes diabéticos. Material y métodos: Estudio de cohortes retrospectivo, a partir de pacientes incluidos en el registro ARIAM-SEMICYUC, con el diagnostico al ingreso de SCA y datos de mortalidad hospitalaria. Se valora el poder pronóstico del score GRACE mediante el cálculo del área bajo la curva ROC, la calibración del modelo y su aplicabilidad clínica en función del tipo de SCA, y de la presencia o no de DM. Por último se estudia el valor añadido de la variable DM en el score mediante la mejora neta de reclasificación.
\end{abstract}

Resultados: El score GRACE muestra un adecuado poder predictivo para mortalidad hospitalaria en la población de estudio, con un grado moderado de calibración, sin diferencias significativas ni en función del tipo de SCA ni en función de la presencia o no de DM. La variable DM no añade valor pronóstico al score.

Conclusiones: La escala GRACE tiene un adecuado poder pronóstico, con buena calibración y buena aplicabilidad clínica en el subgrupo de pacientes diabéticos. 


\section{INTRODUCCIÓN:}

La estratificación del riesgo en el manejo inicial de los pacientes con SCA está recomendado en las principales guías clínicas, y es ampliamente utilizado en la práctica clínica habitual, siendo los principales scores utilizados, el CRUSADE, TIMI y GRACE $^{[1][2][3][4][5]}$.

Por otro lado, la diabetes mellitus es una variable asociada de forma independiente con la mortalidad de los pacientes con $\mathrm{SCA}^{[6][7]}$, por lo que parece lógico que esté incluida en estos scores, como así es en el caso del TIMI y CRUSADE, pero no del GRACE, el cual no incluye esta variable.

La escala GRACE es un modelo multivariable de pronostico que predice mortalidad hospitalaria, y que se aplica de la misma forma en todos los pacientes con SCA, tengan o no elevación del segmento $\mathrm{ST}^{[5]}$. Granger et al. desarrollaron un primer modelo predictivo con 15 variables entre las que se incluía la diabetes mellitus, ya que en el subgrupo de pacientes diabéticos existían diferencias estadísticamente significativas respecto a la mortalidad hospitalaria. A partir de este modelo inicial se deriva un modelo simplificado con mayor utilidad clínica, que incluye únicamente 8 variables, excluyendo finalmente la DM. Granger et al. justifican la simplificación del modelo ya que su poder predictivo es muy similar (aunque ligeramente inferior) al del modelo inicial.

Desde el desarrollo del score GRACE, el manejo de los pacientes con SCA ha evolucionado mucho, tanto desde el punto de vista del diagnóstico como a nivel terapéutico. Además, la población actual es probablemente muy diferente a la de la población de origen, con más de 10 años de diferencia. A pesar de todos estos avances, el pronostico de los pacientes diabéticos sigue siendo significativamente peor ${ }^{[8][9]}$.

Este estudio tiene como objetivos: 1) conocer si el score GRACE es aplicable en nuestra población española actual, 2) estudiar la aplicabilidad clínica de este score en el subgrupo de pacientes diabéticos, y 3) valorar si la inclusión de la variable DM en el modelo puede mejorar el poder pronóstico del mismo.

\section{PACIENTES Y MÉTODO:}

Se trata de un estudio observacional retrospectivo, basado en los datos del registro ARIAM-SEMICIUC, en el que participan diferentes hospitales españoles (63 hospitales en 2015). Se han analizado los datos de aquellos pacientes consecutivos ingresados en la unidad de cuidados intensivos/coronarias con diagnóstico de SCA (con 
y sin elevación del segmento ST), desde el 1 de enero del 2012 hasta el 31 agosto del 2015, y de los que se disponen datos de mortalidad hospitalaria. Los datos han sido analizados con Stata (version 13.1, Stata Corp, College Station, Texas, USA) y el paquete de $\mathrm{R}$ diseñado para la evaluación de modelos de predicción de riesgo PredictABEL.

Se realiza inicialmente un análisis descriptivo que incluye las variables incluidas en el score GRACE, además de la tasa de reperfusión y la mortalidad hospitalaria, comparando los pacientes con y sin DM, en cada uno de los tipos de SCA. Las variables cuantitativas continuas se expresan como la mediana y el rango interquantil, y las variables categóricas como proporciones. La comparación de variables categóricas se realiza con el Test ji al cuadrado y la comparación de las variables cuantitativas mediante el test de Mann-Whitney tras objetivar que dichas variables no siguen una distribución normal mediante la prueba de Shapiro-Wilks. El análisis de contraste se realiza de forma bilateral con un nivel de significación alfa del $5 \%$.

En segundo lugar se valora el poder pronóstico de la escala GRACE mediante el cálculo del área bajo la curva ROC (AUC) en la población de estudio.

Para estudiar la aplicabilidad clínica del score (capacidad del score para predecir la mortalidad hospitalaria en diferentes subgrupos de pacientes), se calcula y se compara el área bajo la curva ROC (AUC) en los diferentes subgrupos, en este caso, en función del tipo de SCA, y en función de la presencia o no de DM.

La calibración del score se refiere al grado de concordancia entre la mortalidad predicha por el mismo y la mortalidad real observada en la población, valorada mediante un modelo de regresión lineal. Se calculan además otros parámetros de calibración del modelo como son la bondad de ajuste de Hosmer-Lemeshow, el score Brier y el estadístico Nagelkerke's R2 ${ }^{[10]}$.

Por último, el efecto de la inclusión de la variable DM en el modelo, se valora mediante el cálculo de Mejora Neta de Reclasificación (NRI, net reclassification indices) en la población general ${ }^{[11]}$.

\section{RESULTADOS:}

Se han analizado un total de 9781 pacientes, 4598 pacientes con SCASEST y 5183 pacientes con SCACEST. La prevalencia de la DM en el grupo de pacientes con SCASEST es de 36,29\% frente a un 24,98\% en el grupo con SCACEST. 
La Tabla 1 muestra las características basales de nuestra población respecto a las variables incluidas en el score GRACE, en función del tipo de SCA y comparando los pacientes con y sin DM.

Tabla 1. Diferencias entre los pacientes con y sin DM, en función del tipo de SCA (Variables incluidas en las escala GRACE, Tasa de reperfusión y Mortalidad hospitalaria por todas las causas)

\begin{tabular}{|c|c|c|c|}
\hline SCASEST & DM $(n=1657)$ & No DM (n=2909) & $\mathbf{p}$ \\
\hline Edad & $72(64-79)$ & $67(56-77)$ & $<0,0001$ \\
\hline TAS (mmHg) & $140(122-160)$ & $135(120-152)$ & $<0,0001$ \\
\hline FC (lpm) & $80(69-95)$ & $75(64-86)$ & $<0,0001$ \\
\hline Cr basal (mg/dl) & $1,05(0,81-1,4)$ & $0,9(0,79-1,1)$ & $<0,0001$ \\
\hline $\begin{array}{l}\text { Elevación enzimas } \\
\text { miocárdicas }\end{array}$ & $91,2 \%$ & $90,55 \%$ & 0,468 \\
\hline Desviación ST & $39,29 \%$ & $37,92 \%$ & 0,011 \\
\hline Paraca cardiaca al ingreso & $1,81 \%$ & $1,82 \%$ & 0,376 \\
\hline $\mathrm{KK}>\mathrm{II}$ & $17,62 \%$ & $6,67 \%$ & $<0,0001$ \\
\hline Reperfusión & $52,96 \%$ & $58,98 \%$ & $<0,0001$ \\
\hline Mortalidad hospitalaria & $7,54 \%$ & $3,92 \%$ & $<0,0001$ \\
\hline SCACEST & $\operatorname{DM}(n=1285)$ & No $\operatorname{DM}(n=3859)$ & $\mathbf{p}$ \\
\hline Edad & $69(60-78)$ & $61(52-74)$ & $<0,0001$ \\
\hline TAS (mmHg) & $130(110-150)$ & $130(110-150)$ & 0,2376 \\
\hline FC (lpm) & $80(67-91)$ & $75(65-88)$ & $<0,0001$ \\
\hline Cr basal (mg/dl) & $0,95(0,8-1,2)$ & $0,9(0,78-1,1)$ & $<0,0001$ \\
\hline $\begin{array}{l}\text { Elevación enzimas } \\
\text { miocárdicas }\end{array}$ & $96,14 \%$ & $97,51 \%$ & 0,011 \\
\hline Paraca cardiaca al ingreso & $4,28 \%$ & $6,09 \%$ & $<0,0001$ \\
\hline $\mathrm{KK}>\mathrm{II}$ & $13,77 \%$ & $7,75 \%$ & $<0,0001$ \\
\hline Reperfusión & $77,74 \%$ & $84,58 \%$ & $<0,0001$ \\
\hline Mortalidad hospitalaria & $10,97 \%$ & $6,01 \%$ & $<0,0001$ \\
\hline
\end{tabular}




\section{Exactitud pronóstica del modelo:}

Para la valoración de la exactitud pronóstica calculamos el área bajo la curva del modelo GRACE para mortalidad hospitalaria (Figura 1), siendo el estadístico C de 0.873 (IC 95\% 0.866 a 0.879 ).

Figura 1: Exactitud pronóstica de la escala GRACE para mortalidad hospitalaria en la población general.

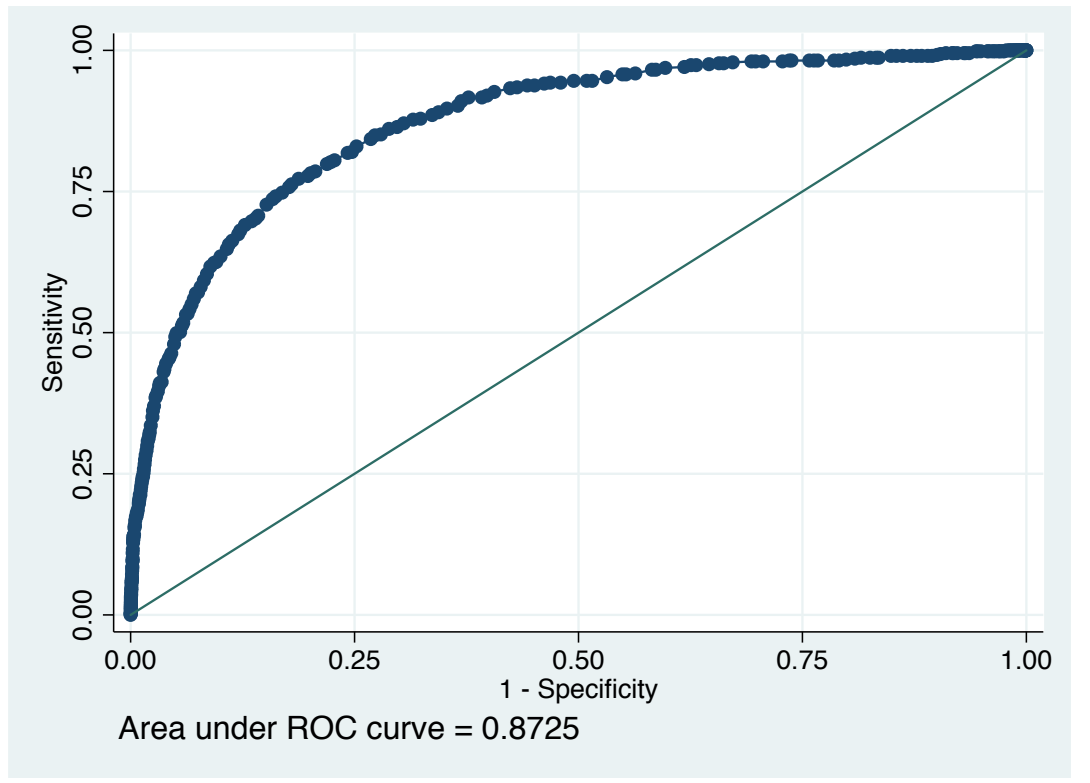

\section{Calibración del modelo:}

En primer lugar, realizamos un modelo de regresión lineal. Para esto, dividimos la puntuación GRACE en 21 tramos similares al del estudio original de Granger et al. y calculamos el porcentaje de mortalidad real observada en nuestra población en cada uno de los tramos. El modelo de regresión se genera al comparar esta mortalidad observada con la mortalidad predicha por el modelo, es decir, la mortalidad en el estudio original, tal y como muestra la Tabla 2. Al realizar el modelo de regresión se objetiva concordancia entre los resultados predichos y observados (Figura 2), con un coeficiente de correlación (r) de 0.99 (IC 95\%: 0.98 to 0.99). 
Tabla 2: Mortalidad predicha por GRACE según Granger et al, comparado con mortalidad observada en nuestra población de estudio en cada uno de los estratos.

\begin{tabular}{|c|c|c|}
\hline Puntuación score GRACE & Mortalidad predicha (\%) & Mortalidad observada (\%) \\
\hline$<60$ & 0,2 & 0 \\
\hline $61-70$ & 0,3 & 0,68 \\
\hline $71-80$ & 0,4 & 0 \\
\hline $81-90$ & 0,6 & 0,96 \\
\hline $91-100$ & 0,8 & 0,48 \\
\hline $101-110$ & 1,1 & 0,35 \\
\hline $111-120$ & 1,6 & 0,51 \\
\hline $121-130$ & 2,1 & 1,58 \\
\hline $131-140$ & 2,9 & 1,19 \\
\hline $141-150$ & 3,9 & 3,49 \\
\hline $151-160$ & 5,4 & 5,21 \\
\hline $161-170$ & 7,3 & 6,1 \\
\hline $171-180$ & 9,8 & 9,81 \\
\hline $181-190$ & 13 & 13,59 \\
\hline $191-200$ & 18 & 18,29 \\
\hline $201-210$ & 23 & 24,31 \\
\hline $211-220$ & 29 & 34,76 \\
\hline $221-230$ & 36 & 40,38 \\
\hline $231-240$ & 44 & 43,28 \\
\hline$>240$ & 52 & 58,82 \\
\hline
\end{tabular}

Figura 2: Modelo de regresión lineal entre la mortalidad predicha y la mortalidad observada.

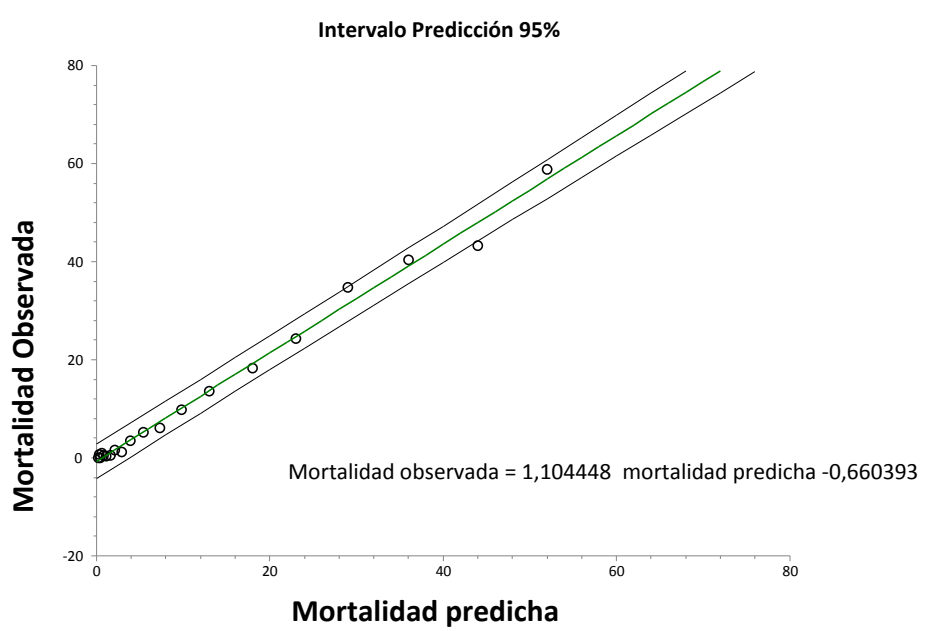

Por otro lado, al calcular el resto de parámetros para valorar la calibración del modelo, objetivamos que esta es al menos moderada, ya que a pesar de que el test de bondad de ajuste de Hosmer-Lemeshow muestra diferencias significativas con $\mathrm{p}=$ 0.0218 , entre los valores observados y los predichos, el score de Brier es de 0.044 (para un modelo de ajuste perfecto los valores deben estar entre 0 y 0,25$)$ y el estadístico R2 
Nagelkerke de 0.34 (su valor puede ir de 0 a 1 , siendo el mejor ajuste cuanto más alto sea el valor $)^{[10][12]}$.

\section{Aplicabilidad clínica:}

Se trata de valorar el poder pronóstico del modelo en los diferentes subgrupos de estudio, para lo cual hemos calculado el área bajo la curva del de la escala GRACE aplicada en la población ARIAM con y sin elevación del ST, y con y sin DM.

Al analizar el poder pronóstico en los pacientes con y sin elevación del segmento ST, objetivamos que en ambos casos el score tiene un valor predictivo para mortalidad hospitalaria adecuado, sin encontrar diferencias significativas entre estos grupos, con $\mathrm{p}=0,2684$, tal y como muestra la figura 3 .

Figura 3: Comparación del poder predictivo del GRACE para mortalidad hospitalaria en la población general en función del tipo de SCA.

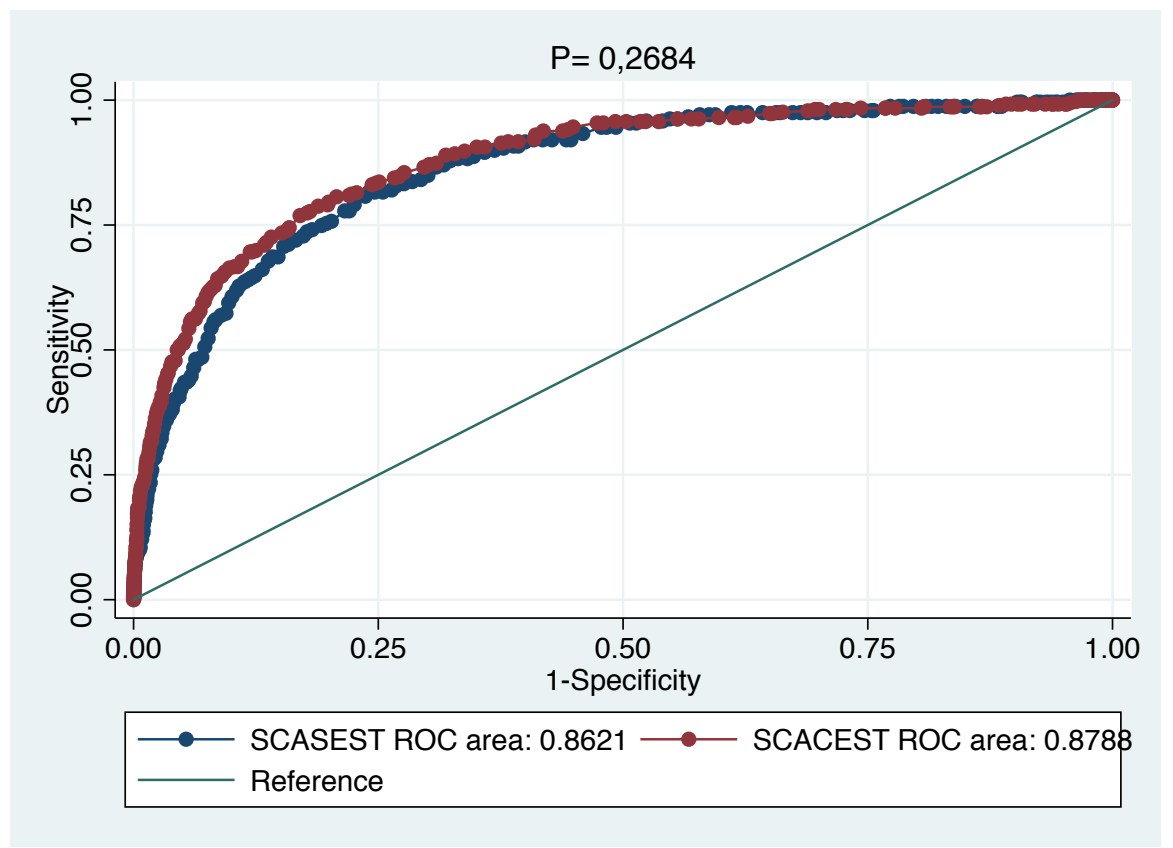

Por otro lado, tampoco se objetivan diferencias significativas al comparar el poder predictivo del score en los pacientes diabéticos frente a los no diabéticos. En el grupo de pacientes con SCASEST con $\mathrm{p}=0,0804$ (Figura 4), y en el grupo con SCACEST, la p es de 0,2113 (Figura 5). 
Figura 4: Comparación del poder predictivo del GRACE para mortalidad hospitalaria en pacientes con SCASEST, con y sin diabetes méllitus. No se objetivan diferencias estadísticamente significativas con $p=$ 0.0804

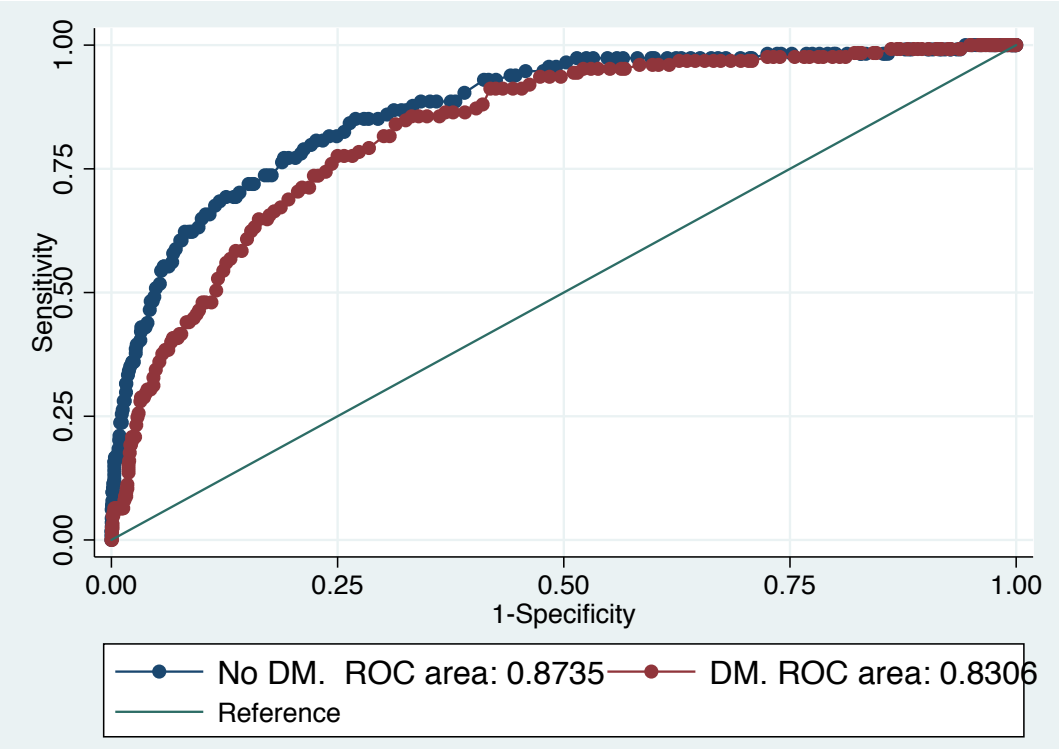

Figura 5. Comparación del poder predictivo del GRACE para mortalidad hospitalaria en pacientes con SCACEST, con y sin diabetes méllitus. No se objetivan diferencias estadísticamente significativas con p = 0,2113

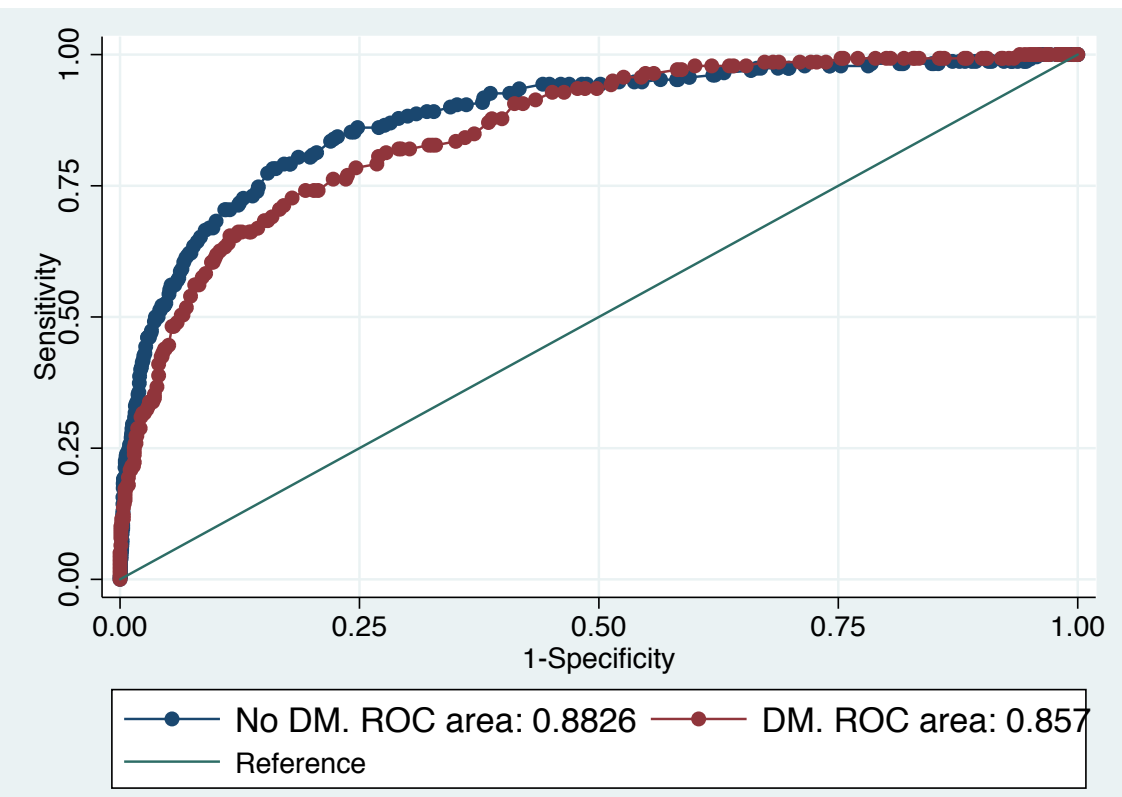




\section{Valor añadido de la DM:}

Para valorar si la inclusión de la variable DM al modelo GRACE mejora la exactitud pronóstica del mismo, se calcula la Mejora Neta de Reclasificación (NRI, net reclassification indices), siendo este de 0,02 ( IC 95\% -0,0108 a 0,0706). Al añadir la variable DM, el nuevo modelo, podemos calcular cuantos pacientes de reclasifican de forma correcta e incorrecta, en función de la presencia del evento (Mortalidad hospitalaria). En el grupo de pacientes que presentan el evento, el nuevo modelo reclasifica de forma correcta un $15,87 \%$ de los pacientes, pero de forma incorrecta a un 7,38\%. En el grupo de los pacientes que no presentan el evento (que no fallecen), el nuevo modelo sólo es capaz de reclasificar de forma correcta un 3,6\% de los pacientes, frente a un $9,1 \%$ que reclasifica de forma incorrecto. Con todos estos datos, podemos afirmar que la variable DM no añade valor pronóstico al score GRACE.

\section{DISCUSIÓN:}

La escala de riesgo isquémico GRACE continua teniendo un adecuado poder predictivo para mortalidad hospitalaria por todas las causas, tanto en los pacientes con SCASEST como con SCACEST, a pesar de los importantes cambios respecto al manejo de estos pacientes desde que el score fue creado $^{[13]}$, tal y como hemos podido objetivar en nuestro estudio. Estos hallazgos confirman el resultado de estudios previos que también han estudiado la validación de la escala GRACE, en diferentes poblaciones contemporáneas, obteniendo resultados similares ${ }^{[14][15][16]}$.

Podemos afirmar además que el grado de calibración del score en nuestra población es al menos moderado. El score de brier de 0,04 muestra un ajuste casi perfecto (modelo perfecto de 0 a 0,25), y el valor de R2 de Nagelkerke de 0,34 indica ajuste moderado (sus valores van de 0 a 1 , siendo mejor el ajuste cuanto más alto es el valor). El test de bondad de ajuste de Hosmer-Lemeshow muestra diferencias significativas entre los valores predichos y los observados con una $\mathrm{p}$ de 0,02 , sin embargo no aporta realmente información sobre la falta de calibración, no siendo recomendable su uso para valorar este parámetro, especialmente en muestras grandes como la nuestra ${ }^{[12]}$.

Por otro lado, la aplicabilidad clínica del score en diferentes subgrupos de pacientes de alto riesgo, como son los pacientes diabéticos, ha sido poco estudiado. Además, en un estudio previo hemos podido objetivar que la gravedad de los pacientes diabéticos medida por GRACE puede estar asociada de forma independiente con la 
mortalidad hospitalaria de los mismo ${ }^{[17]}$, por lo que consideramos de gran importancia poder comprobar la validez de dicho escore en estos pacientes.

En nuestro estudio el score GRACE tiene un poder predictivo similar en pacientes con y sin diabetes mellitus, confirmando resultados de estudios previos ${ }^{[18]}, \mathrm{y}$ justifica por lo tanto, la decisión de Granger et al. de excluir esta variable del score final $^{[5]}$. Tampoco hemos podido objetivar que el añadir la variable DM al modelo, mejore su capacidad predictiva.

Este estudio analiza una muestra muy representativa de la población española actual, ya que el registro ARIAM-SEMICYUC es un registro multicéntrico en el que participan centros de toda España . Sin embargo, al ser un registro de base hospitalaria, sólo se incluyen los pacientes que ingresan en las unidades coronarias, por lo que los resultados del estudio pueden verse sesgados por la pérdida de aquellos pacientes que ingresan directamente en planta o que fallecen antes de llegar al hospital. Por otro lado, las pérdidas derivadas de la variable mortalidad hospitalaria. De la misma manera, existe un porcentaje de pérdidas en la variable mortalidad hospitalaria que, dado que son pérdidas aleatorias, consideramos que no tiene porqué sesgar nuestros resultados.

Parece por tanto, que la utilización del GRACE como escala de riesgo isquémico, tal y como recomiendan las guías, es adecuado, tanto en la población española actual como en el subgrupo de pacientes diabéticos. 


\section{BIBLIOGRAFÍA:}

1. Subherwal S, Bach RG, Chen AY, Gage BF, Rao S V, Newby LK, et al. Baseline risk of major bleeding in non-ST-segment-elevation myocardial infarction: the CRUSADE (Can Rapid risk stratification of Unstable angina patients Suppress ADverse outcomes with Early implementation of the ACC/AHA Guidelines) Bleeding Score. Circulation 2009;119(14):1873-82.

2. Kadakia MB, Desai NR, Alexander KP, Chen AY, Foody JM, Cannon CP, et al. Use of anticoagulant agents and risk of bleeding among patients admitted with myocardial infarction: a report from the NCDR ACTION Registry--GWTG (National Cardiovascular Data Registry Acute Coronary Treatment and Intervention Outcomes Network Registry--G. JACC Cardiovasc Interv 2010;3(11):1166-77.

3. Antman EM, Cohen M, Bernink PJ, McCabe CH, Horacek T, Papuchis G, et al. The TIMI risk score for unstable angina/non-ST elevation MI: A method for prognostication and therapeutic decision making. JAMA 2000;284(7):835-42.

4. Morrow DA, Antman EM, Charlesworth A, Cairns R, Murphy SA, de Lemos JA, et al. TIMI risk score for ST-elevation myocardial infarction: A convenient, bedside, clinical score for risk assessment at presentation: An intravenous nPA for treatment of infarcting myocardium early II trial substudy. Circulation 2000;102(17):2031-7.

5. Granger CB, Goldberg RJ, Dabbous O, Pieper KS, Eagle KA, Cannon CP, et al. Predictors of hospital mortality in the global registry of acute coronary events. Arch Intern Med 2003;163(19):2345-53.

6. Donahoe SM, Stewart GC, McCabe CH, Mohanavelu S, Murphy SA, Cannon $\mathrm{CP}$, et al. Diabetes and mortality following acute coronary syndromes. JAMA 2007;298(7):765-75.

7. Hasin T, Hochadel M, Gitt AK, Behar S, Bueno H, Hasin Y. Comparison of treatment and outcome of acute coronary syndrome in patients with versus patients without diabetes mellitus. Am J Cardiol 2009;103(6):772-8.

8. Malmberg K, Yusuf S, Gerstein HC, Brown J, Zhao F, Hunt D, et al. Impact of diabetes on long-term prognosis in patients with unstable angina and non-Q-wave myocardial infarction: results of the OASIS (Organization to Assess Strategies for Ischemic Syndromes) Registry. Circulation 2000;102(9):1014-9.

9. Franklin K, Goldberg RJ, Spencer F, Klein W, Budaj A, Brieger D, et al. 
Implications of diabetes in patients with acute coronary syndromes. The Global Registry of Acute Coronary Events. Arch Intern Med 2004;164(13):1457-63.

10. Steyerberg EW, Vickers AJ, Cook NR, Gerds T, Gonen M, Obuchowski N, et al. Assessing the performance of prediction models: a framework for traditional and novel measures. Epidemiology 2010;21(1):128-38.

11. Kerr KF, Wang Z, Janes H, McClelland RL, Psaty BM, Pepe MS. Net reclassification indices for evaluating risk prediction instruments: a critical review. Epidemiology 2014;25(1):114-21.

12. Steyerberg EW, Vergouwe Y. Towards better clinical prediction models: seven steps for development and an ABCD for validation. Eur Heart $J$ 2014;35(29):1925-31.

13. Fox KAA, Steg PG, Eagle KA, Goodman SG, Anderson FA, Granger CB, et al. Decline in rates of death and heart failure in acute coronary syndromes, 19992006. JAMA 2007;297(17):1892-900.

14. Elbarouni B, Goodman SG, Yan RT, Welsh RC, Kornder JM, Deyoung JP, et al. Validation of the Global Registry of Acute Coronary Event (GRACE) risk score for in-hospital mortality in patients with acute coronary syndrome in Canada. Am Heart J 2009;158(3):392-9.

15. Fox KAA, Fitzgerald G, Puymirat E, Huang W, Carruthers K, Simon T, et al. Should patients with acute coronary disease be stratified for management according to their risk? Derivation, external validation and outcomes using the updated GRACE risk score. BMJ Open 2014;4(2):e004425.

16. Shaikh MK, Hanif B, Shaikh K, Khan W, Parkash J. Validation of Grace Risk Score in predicting in-hospital mortality in patients with non ST-elevation myocardial infarction and unstable angina. J Pak Med Assoc 2014;64(7):807-11.

17. Baeza-Román A, de Miguel-Balsa E, Latour-Pérez J, Díaz de Antoñana-Saez V, Arguedas-Cervera J, Mira-Sánchez E, et al. Accessibility to health care of diabetic patients with acute coronary syndrome ST-segment elevation. Med Intensiva 2016;40(2):90-5.

18. Abu-Assi E, Ferreira-González I, Ribera A, Marsal JR, Cascant P, Heras M, et al. «Do GRACE (Global Registry of Acute Coronary events) risk scores still maintain their performance for predicting mortality in the era of contemporary management of acute coronary syndromes?». Am Heart J 2010;160(5):826-8343. 